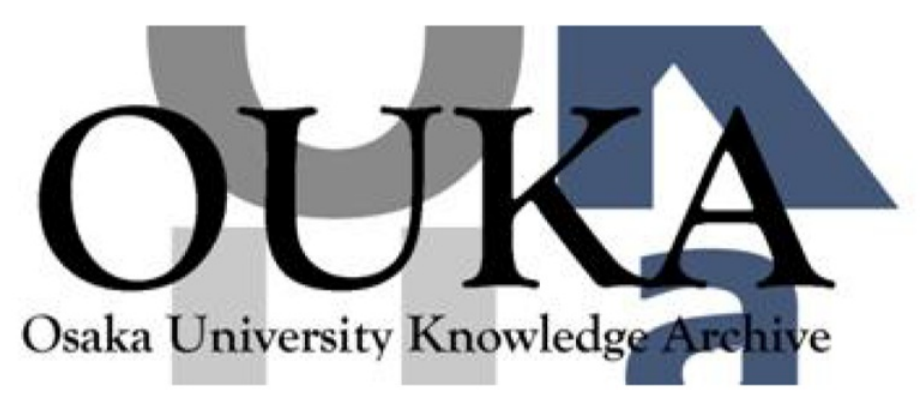

\begin{tabular}{|c|c|}
\hline Title & $\begin{array}{l}\text { Zero-multipole summation method for efficiently } \\
\text { estimating electrostatic interactions in } \\
\text { molecular system }\end{array}$ \\
\hline Author (s) & Fukuda, Ikuo \\
\hline Citation & The Journal of Chemical Physics. 139 p. 174107 \\
\hline Issue Date & 2013 \\
\hline oaire:version & VoR \\
\hline URL & https://hdl. handle. net/11094/52403 \\
\hline rights & $\begin{array}{l}\text { Copyright (2013) American Institute of Physics. } \\
\text { This article may be downloaded for personal use } \\
\text { only. Any other use requires prior permission } \\
\text { of the author and the American Institute of } \\
\text { Physics. }\end{array}$ \\
\hline Note & \\
\hline
\end{tabular}

Osaka University Knowledge Archive : OUKA

https://ir. Library. osaka-u. ac. jp/

Osaka University 


\section{A The Journal of Chemical Physics}

\section{Zero-multipole summation method for efficiently estimating electrostatic interactions in molecular system}

Ikuo Fukuda

Citation: The Journal of Chemical Physics 139, 174107 (2013); doi: 10.1063/1.4827055

View online: http://dx.doi.org/10.1063/1.4827055

View Table of Contents: http://scitation.aip.org/content/aip/journal/jcp/139/17?ver=pdfcov

Published by the AIP Publishing

\section{Articles you may be interested in}

Bézier curve string method for the study of rare events in complex chemical systems

J. Chem. Phys. 141, 074110 (2014); 10.1063/1.4893216

The zero-multipole summation method for estimating electrostatic interactions in molecular dynamics: Analysis of the accuracy and application to liquid systems

J. Chem. Phys. 140, 194307 (2014); 10.1063/1.4875693

Notes on "Ewald summation of electrostatic multipole interactions up to quadrupolar level" [J. Chem. Phys.119, 7471 (2003)]

J. Chem. Phys. 129, 074102 (2008); 10.1063/1.2970887

Optimal charge-shaping functions for the particle-particle-particle-mesh (P3M) method for computing electrostatic interactions in molecular simulations

J. Chem. Phys. 113, 10464 (2000); 10.1063/1.1324713

An Ewald summation based multipole method

J. Chem. Phys. 113, 3492 (2000); 10.1063/1.1289918

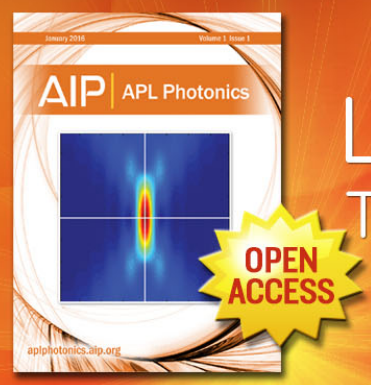

Launching in 2016! The future of applied photonics research is here 


\title{
Zero-multipole summation method for efficiently estimating electrostatic interactions in molecular system
}

\author{
Ikuo Fukuda \\ Institute for Protein Research, Osaka University, 3-2 Yamadaoka, Suita, Osaka 565-0871, Japan and \\ RIKEN (The Institute of Physical and Chemical Research), 2-1 Hirosawa, Wako, Saitama 351-0198, Japan
}

(Received 11 July 2013; accepted 14 October 2013; published online 5 November 2013)

\begin{abstract}
The zero-multipole summation method has been developed to efficiently evaluate the electrostatic Coulombic interactions of a point charge system. This summation prevents the electrically nonneutral multipole states that may artificially be generated by a simple cutoff truncation, which often causes large amounts of energetic noise and significant artifacts. The resulting energy function is represented by a constant term plus a simple pairwise summation, using a damped or undamped Coulombic pair potential function along with a polynomial of the distance between each particle pair. Thus, the implementation is straightforward and enables facile applications to high-performance computations. Any higher-order multipole moment can be taken into account in the neutrality principle, and it only affects the degree and coefficients of the polynomial and the constant term. The lowest and second moments correspond respectively to the Wolf zero-charge scheme and the zero-dipole summation scheme, which was previously proposed. Relationships with other non-Ewald methods are discussed, to validate the current method in their contexts. Good numerical efficiencies were easily obtained in the evaluation of Madelung constants of sodium chloride and cesium chloride crystals. (C) 2013 AIP Publishing LLC. [http://dx.doi.org/10.1063/1.4827055]
\end{abstract}

\section{INTRODUCTION}

Molecular simulation with molecular dynamics (MD) and Monte-Carlo (MC) calculations is now widely used to analyze various macroscopic systems, such as condensed matter and bimolecular systems, with microscopic descriptions. In these simulations, appropriate treatment of the electrostatic interactions among the charged particles in the system is critical, because the interactions are essential for various phenomena in a number of systems. The treatment should be accurate, and the simple truncated evaluation cannot be used, since the interactions are long-range. ${ }^{1}$

One of the representative approaches is the lattice sum (LS) method, such as the Ewald method, ${ }^{2}$ when we assume the periodic boundary condition (PBC). In the threedimensional PBC, the sum of the Coulombic interactions $\phi_{i j}\left(r_{i j}\right) \equiv q_{i} q_{j} / r_{i j}$ for charges $q_{i}$ and $q_{j}$ with their distance $r_{i j}$ is conditionally convergent in general, and its heuristic ordering in the summation often fails (the cubic-expanding sum converges, but the sphere-expanding sum diverges). ${ }^{3}$ The Ewald energy plus the dipolar term is interpreted to be the value of the sum obtained by spherical-shell ordering with respect to the copies of the original unit cell, ${ }^{4}$ and the Ewald sum holds if the crystal is supposed to be immersed in a conductor which cancels the dipolar term. The Ewald method, with the aid of computationally efficient devices including the mesh-based approach, ${ }^{5}$ has been traditionally used in computer simulations. With developments facilitated by several pioneering works, ${ }^{6-8}$ other types of formulae have been studied. By evaluating Fourier expansions in modified Bessel functions, Hautot ${ }^{9}$ led series using hyperbolic functions for Madelung constants. Lekner ${ }^{10}$ utilized Bessel functions of imaginary arguments and derived rapidly convergent sums.
Tyagi ${ }^{11}$ derived a fast convergent method, which does not include optimizing parameters such as the damping parameter in the Ewald sum, and arrived at very accurate Madelung energies. ${ }^{12,13}$ Note that the main focus in the application of these approaches is the efficient expression of the type $\sum_{n \in \mathbb{Z}^{d}} \phi_{i j}\left(\left\|x_{i}-x_{j}-L n\right\|\right)$, for arbitrarily fixed particle coordinates $x_{i}$ and $x_{j} \in \mathbb{R}^{d}$ (if $i=j$, one uses $\mathbb{Z}^{d} \backslash\{0\}$ instead of $\mathbb{Z}^{d}$ ). However, for actual molecular simulations that often treat general disordered systems, we should also consider the efficiency in evaluating the above summation with respect to all pairs of $i$ and $j$.

The PBC is required in crystal analyses, and it is also useful to simulate a bulk state of matter since it allows us to avoid the creation of an interface, which often causes significant artifacts in simulations. However, in some cases, the application of the PBC is not appropriate, such as in an intrinsically non-periodic system. ${ }^{14}$ Thus, the consideration of other approaches would be valuable.

When conducting molecular simulations, we cannot avoid the issue of the computational costs to evaluate the electrostatic interactions. In fact, almost all computational costs are for the evaluation of long-range interactions in classical MD and MC simulations. This cost is proportional to the square of the number of particles in a target classical system, and would become greater if we consider quantum effects. This is a bottleneck for simulating a large system, which is required to prepare a realistic physical model to investigate physical, chemical, and biological phenomena in detail.

From this computational viewpoint, a cutoff-based (CB) approach is appealing, since it would provide an $O(N)$ scheme for a larger system. In addition, CB methods are useful since they are irrelevant to the boundary conditions, in general. In 
such a CB approach, often called a non-Ewald approach, ${ }^{14}$ the reaction field (RF) method ${ }^{15,16}$ assumes that the region outside the cutoff sphere is a dielectric continuum polarized by reacting with the molecules inside the cavity. An electric field generated by this polarization interacts with the dipoles in the sphere, adding another term to the Coulombic form. ${ }^{17-20}$ The pre-averaging method ${ }^{21}$ was introduced by Yakub and Ronchi, to remove the artificial cubic symmetry in the LS method, and the energy formula was obtained using the Ewald summation expansion and by averaging the quantities over spherical angular coordinates in the expansion. ${ }^{21-24}$ In this context, but based on the application of Gauss's law, a spherically averaged periodic potential was recently obtained. ${ }^{25}$ The isotropic periodic summation by $\mathrm{Wu}$ and Brooks ${ }^{26}$ assumes the isotropic periodicity, rather than the cubic periodicity, and has been developed for polar systems ${ }^{27}$ and for further applications. ${ }^{28-30} \mathrm{Wu}$ and Brooks proposed different versions of their isotropic periodic sum method for homogeneous, heterogeneous, and finite systems. ${ }^{31}$ Wolf et al. ${ }^{32,33}$ provided a simple and crucial physical perspective to the potential-shift truncation approach, in which the charge neutrality is important to achieve accurate total energy and the potential-function shifting procedure corresponds to the charge neutralization procedure (except the self term), which can be attained by placing the image charges at the cutoff surface.

This zero-charge (ZC) scheme by Wolf et al. ${ }^{33}$ is simple enough, and many applications and developments have followed, including efforts by Zahn, Schilling, and Kast, ${ }^{34}$ Avendaño and Gil-Villegas, ${ }^{35}$ Fennell and Gezelter, ${ }^{36}$ and others. $^{37-55}$ In developing the ZC principle, ${ }^{56,57}$ the zerodipole (ZD) summation method ${ }^{58}$ provides the energy derived by counting the interactions for a neutralized subset, regarding the dipoles as well as the charges. The applications of the ZD method to several systems ${ }^{58-60}$ have shown that it can provide more accurate electrostatic energies than the $\mathrm{ZC}$ scheme.

In this paper, we develop this idea further to reach a more accurate scheme, using a higher order expansion of the pair potential function. This scheme addresses to the point that the summation over a certain subset for which the total multipole moments yields zero should be taken for an individual particle, in order to attain the total energy in an effective manner. The current formalism provides a more accurate pairwise sum expression of the excess energy, as compared with the ZD method, as long as the physical states allow the description of the zero-multipole ( $\mathrm{ZM})$ principle. This is because more information about the charge arrangement for the physical states is included in the newly developed energy functional. The purpose is to develop a method appropriate for both periodic and non-periodic systems, and to construct a very simple algorithm that is readily applicable to any computational architecture.

Section II first introduces the background of the method and describes the physical motivation for considering the current zero-multipole summation method, followed by a simple illustration of the main result of this section. Then, after the details of the notions of the zero-multipole state and a technical view of the zero-multipole summation are provided, we see that the excess energy representation is critical to construct a numerically applicable scheme. To obtain this, two strategies are employed: one for a specific analytical approximation of the potential function on a relevant domain, and the other for a simple algebraic representation towards the final summation form. In Sec. III, we investigate the functional form of the ZM energy and the properties of the pair potential function, and analyze the errors included in the method. We place the current method in the context of related methods, and provide a unified view among them. The results of simple numerical studies are shown, and we investigate the efficiency of the method, using typical crystal states. Section IV concludes with remarks.

\section{ZERO-MULTIPOLE SUMMATION}

\section{A. Main result}

The main result obtained in this section is an approximate formula of the summation $\sum_{i, j} q_{i} q_{j} / r_{i j}$. The formula is represented by

$$
\frac{1}{2} \sum_{i} \sum_{\substack{j(\neq \neq i) \\ r_{i j}<r_{\mathrm{c}}}} q_{i} q_{j}\left[1 / r_{i j}-\sum_{m=0}^{l} a_{m}^{(l)} r_{i j}^{2 m}\right]-\frac{a_{0}^{(l)}}{2} \sum_{i} q_{i}^{2},
$$

where $r_{\mathrm{c}}$ is the cutoff length and the second term in the pairwise sum is a polynomial for which the coefficients $\left\{a_{0}^{(l)}, \ldots a_{l}^{(l)}\right\}$ are determined in a certain unique manner.

The general idea and the outline of the derivation are as follows. Having chosen a cutoff sphere, it is asserted on physical grounds that for every $i$ the particles $j$ in $i$ 's cutoff sphere almost satisfy a set of zero multipole conditions (zero lthmoment condition). They are split into two subsets: one is a relatively larger subset (termed a zero multipole subset) in which the particles satisfy a zero multipole condition; and the other is a relatively smaller subset (termed an excess subset) in which the particles are contained in a thin shell close to the cutoff sphere surface (see Fig. 1). The potential energy for atom $i$ due to all other atoms should be restricted to those in the larger subset (viz., zero multipole subset). This sum over the larger subset can be written as the standard cutoff sum minus the potential energy due to the smaller subset (viz., excess subset) positioned within the thin shell. The potential energy for particles within this thin shell can be approximated by a polynomial, and by virtue of the zero-multipole conditions, this energy can be approximated by the same polynomial now evaluated over all $j$ in the cutoff sphere. Therefore, although the members of the smaller set are not necessarily known, the total energy can be represented by a standard cutoff sum of the Coulombic potential minus the polynomial, along with a constant. This constant can be regarded as a "self" term [the last term of Eq. (1)]. We can extend formula (1) using a damping factor, and this extended form is described below.

\section{B. Physical motivation}

Let $x \equiv\left(x_{1}, \ldots, x_{N}\right)$ be $N$ particle configurations in a coordinate phase space $\Gamma$, and $q_{j}$ be the charge of particle $j$ that moves in space $\mathbb{R}^{d}$ (the main concern is in $d=3$ ) for $j \in \mathcal{N} \equiv\{1, \ldots, N\}$. We calculate the total Coulombic 


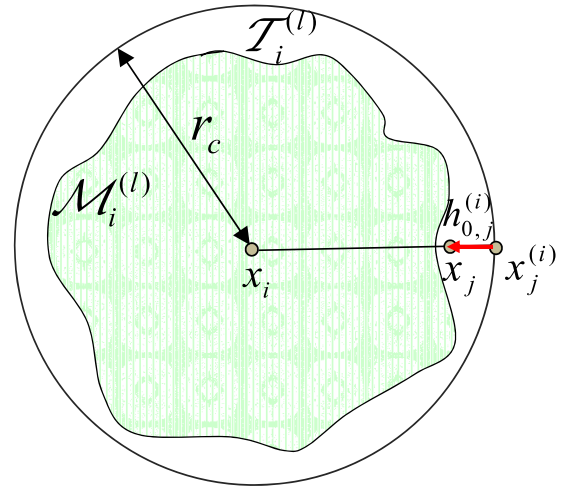

FIG. 1. The conventional simple cutoff method considers all charges in a cutoff sphere with radius $r_{\mathrm{c}}$. The current zero-multipole summation method conceptually deals with the particles only in the shaded region, which schematically represent the zero $l$ th multipole subset $\mathcal{M}_{i}^{(l)}$. The particle in the nonshaded region represents a member of the excess subset $\mathcal{J}_{i}^{(l)}$. Particle $j$ at position $x_{j}$ is one of the members, and $x_{j}^{(i)}$ is the shifted position of $x_{j}$ towards the cutoff surface along the vector $x_{j}-x_{i}$; while $x_{j}^{(i)}=x_{j}$ for a particle belonging to $\mathcal{M}_{i}^{(l)}$.

energy $E(x)$ for $x \in \Gamma$,

$$
\begin{aligned}
E(x) & =\frac{1}{2} \sum_{i \in \mathcal{N}} \sum_{j \in \mathcal{N}_{i}} \frac{q_{i} q_{j}}{r_{i j}} \\
& =\frac{1}{2} \sum_{i \in \mathcal{N}} \sum_{j \in \mathcal{N}_{i}} q_{i} q_{j} V\left(r_{i j}\right)+\frac{1}{2} \sum_{i \in \mathcal{N}} \sum_{j \in \mathcal{N}_{i}} q_{i} q_{j}\left[\frac{1}{r_{i j}}-V\left(r_{i j}\right)\right],
\end{aligned}
$$

where $r_{i j} \equiv\left\|x_{i j}\right\|$ with $x_{i j} \equiv x_{i}-x_{j} \in \mathbb{R}^{d}$ for every $i$ and $j \in \mathcal{N}_{i} \equiv \mathcal{N}-\{i\}$. In this case, the function $V$, used in the decomposition of $1 / r$, is specifically set as

$$
V(r)=\frac{\operatorname{erfc}(\alpha r)}{r}
$$

where $\operatorname{erfc}(\alpha r)$ is the complementary error function of $\alpha r$, and becomes a damping factor for the bare Coulomb potential function as long as we use $\alpha \geq 0$. A relatively large $\alpha$ is used in the conventional Ewald approach, under the PBCs, and its damping effect enables the first term of Eq. (2b) to be evaluated by a simple cutoff truncation. However, with such a large $\alpha$, the evaluation of the second term is not simple, and the key point in the approach is to generate a Fourier expansion. Here, we utilize the opposite aspect of the function, i.e., a small $\alpha$. In this case, the second term of Eq. (2b) can be very simply approximated $^{33,58}$ as

$$
\begin{aligned}
& \frac{1}{2} \sum_{i \in \mathcal{N}} \sum_{j \in \mathcal{N}_{i}} q_{i} q_{j}\left[\frac{1}{r_{i j}}-V\left(r_{i j}\right)\right] \\
& =\frac{1}{2} \sum_{i \in \mathcal{N}} \sum_{j \in \mathcal{N}} \frac{q_{i} q_{j}}{r_{i j}} \operatorname{erf}\left(\alpha r_{i j}\right)-\frac{1}{2} \lim _{r \rightarrow 0} \frac{\operatorname{erf}(\alpha r)}{r} \sum_{i \in \mathcal{N}} q_{i}^{2}, \\
& \sim-\frac{\alpha}{\sqrt{\pi}} \sum_{i \in \mathcal{N}} q_{i}^{2} \quad \text { for small } \alpha .
\end{aligned}
$$

In fact, $\operatorname{erf}(\alpha r)$ decreases along with the value of $\alpha$, and the limit, $\alpha=0$, yields Eq. (4) to be exact. In contrast, the accurate evaluation of the first term of Eq. (2b) becomes nontrivial for such a small $\alpha$, considering the fact that the limit, $\alpha=$ 0 , leads to the bare Coulomb, $V\left(r_{i j}\right)=1 / r_{i j}$, which does not allow a simple truncation.

Together with Nakamura and Yonezawa, in our previous approach, ${ }^{58}$ we considered that for equilibrated configurations observed in, e.g., a physically stable system, the neutrality of both the charges and dipoles is attained even at a certain local level, and this physical information should be taken into account to evaluate the interaction. However, the particle configuration state inside a simply chosen distance-judged cutoff sphere often shows physical instability and generates noise when evaluating the true energy value. Thus, the interactions for an individual particle $i$ should be counted for such a neutralized subset of particles, instead of counting all of the particles in a given cutoff sphere (viz., the neutralized subset does not necessarily form a "complete" sphere). For such a (zerocharge and) zero-dipole state, a summation method that reflects this neutrality condition promises to provide a good approximation to estimate the energy. In fact, the accuracy of the method has been shown in actual numerical simulations, such as ionic systems, ${ }^{58}$ a bulk water system, ${ }^{59}$ and a membrane protein system with explicit membrane and solvent molecules and ions. 60

This neutralization view can be extended to higher-order multipole moments; i.e., we can suppose that the zero $l$ th moment condition holds locally, where local means a sub-area that is not the total, but is sufficiently large in a realistic system. Although the validity of this assumption should be evaluated in individual cases, we can construct a theoretical framework if we admit this as a physical assumption.

\section{Neutralization principle}

With these considerations, the neutralization principle is specifically characterized as follows: for $x \in \Gamma$ and for $i \in \mathcal{N}$, there exists a zero-lth-moment (zero-multipole) subset $\mathcal{M}_{i}^{(l)}$ $\subset \mathcal{N}_{i}$ such that

$$
\begin{aligned}
& \forall j \in \mathcal{M}_{i}^{(l)}, r_{i j}<r_{\mathrm{c}}, \\
& \sum_{j \in \mathcal{M}_{i}^{(l)} \cup\{i\}} q_{j} x_{j} \otimes \stackrel{m}{\cdots} \otimes x_{j}=0 \in \mathbb{R}^{d} \otimes \stackrel{m}{.} \otimes \mathbb{R}^{d} \\
& \text { for } m=0, \ldots, l \text {, } \\
& \forall j \in \mathcal{N}_{i}-\mathcal{M}_{i}^{(l)},\left(r_{i j}<r_{\mathrm{c}} \Rightarrow r_{i j} \simeq r_{\mathrm{c}}\right) .
\end{aligned}
$$

The first condition, Eq. (5a), simply means that all particles in $\mathcal{M}_{i}^{(l)}$ are inside the cutoff sphere with the radius (cutoff length) $r_{\mathrm{c}}>0$. Condition (5b) means that the total $m$ th moment in the neutralized subset adding the target particle $i, \mathcal{M}_{i}^{(l)} \cup\{i\}$, vanishes for all $m \leq l$, viz., zero-multipole conditions. For example, in $l=0$, this is simply the zero-charge condition, $\sum_{j \in \mathcal{M}_{i}^{(0)} \cup\{i\}} q_{j}=0$, which is utilized in the Wolf scheme, ${ }^{33,56}$ the condition with $l=1$ is the (zero-charge and) zero-dipole condition, $\sum_{j \in \mathcal{M}_{i}^{(1)} \cup\{i\}} q_{j}=0 \in \mathbb{R}$ and $\sum_{j \in \mathcal{M}_{i}^{(1)} \cup\{i\}} q_{j} x_{j}=0 \in \mathbb{R}^{d}$, as 
derived in the ZD method. ${ }^{58}$ Similarly, for moment $l$, the neutralized condition is described by order- 0 , order- $1, \ldots$, and order- $l$ tensors. Condition $(5 \mathrm{c})$ means that any particle (except $i$ ) not belonging to $\mathcal{M}_{i}^{(l)}$, but inside the cutoff sphere, is located close to the cutoff surface. In other words, the neutrality should be disrupted near the surface. For simplicity, we often call $x \in \Gamma$ a $l$ th zero-multipole state or the ZM state.

Several remarks are addressed. As discussed in Ref. 58, exactly speaking, the phase space $\Gamma \equiv \Gamma_{l}$ should not simply be the bare phase space $\Gamma_{\mathrm{b}} \equiv\left\{x \in \mathbb{R}^{d N} \mid x_{i} \neq x_{j}\right.$ for $i \neq j$ $(\forall i, j \in \mathcal{N})\}$, but a subset of all $x \in \Gamma_{\mathrm{b}}$ having $\mathcal{M}_{i}^{(l)} \subset \mathcal{N}_{i}$ such that condition (5) holds for any $i \in \mathcal{N}$, instead of assuming that every $x \in \Gamma_{\mathrm{b}}$ has such $\mathcal{M}_{i}^{(l)}$ for all $i$. Although $\mathcal{M}_{i}^{(l)}$ is not necessarily fixed uniquely, the choice does not affect the results, as shown below. Note that $\mathcal{M}_{i}^{(l)}$ depends on $x$, viz., we are considering $\mathcal{M}_{i}^{(l)} \equiv \mathcal{M}_{i}^{(l)}(x)$. For simply conducting an extensive application, it would be better to weaken the conditions by replacing the equalities with near equalities in the conditions $(5 b)$.

Now, in the first term of Eq. (2b), we use the currently introduced strategy, viz., the replacement

$$
\sum_{j \in \mathcal{N}_{i}} q_{j} V\left(r_{i j}\right) \rightarrow \sum_{j \in \mathcal{M}_{i}^{(l)}} q_{j} V\left(r_{i j}\right)
$$

Namely, the only contributions that should be counted are those from the ZM subset for every $i$. In other words, we assume the approximation

$$
\sum_{i \in \mathcal{N}} \sum_{j \in \mathcal{N}_{i}} q_{i} q_{j} V\left(r_{i j}\right) \approx \sum_{i \in \mathcal{N}} \sum_{j \in \mathcal{M}_{i}^{(l)}} q_{i} q_{j} V\left(r_{i j}\right),
$$

where the sum with respect to $\mathcal{M}_{i}^{(l)}$ is called a zero-lth(multi)pole summation, or, in short, zero-multipole (ZM) summation [note that Eq. (7), which adopts the sum with respect to $i$, is a weaker condition than the replacement, Eq. (6), which concerns an individual $i$.] Hence, from Eqs. (2), (4), (5a), and (7), we obtain

$$
E(x) \simeq \frac{1}{2} \sum_{i \in \mathcal{N}} \sum_{\substack{j \in \mathcal{N}_{i} \\ r_{i j}<r_{\mathrm{c}}}} q_{i} q_{j} V\left(r_{i j}\right)-\hat{E}(x)-\frac{\alpha}{\sqrt{\pi}} \sum_{i \in \mathcal{N}} q_{i}^{2},
$$

where

$$
\hat{E}(x) \equiv \hat{E}^{(l)}(x) \equiv \frac{1}{2} \sum_{i \in \mathcal{N}} \sum_{j \in \mathcal{J}_{i}^{(l)}} q_{i} q_{j} V\left(r_{i j}\right),
$$

with $\mathcal{J}_{i}^{(l)} \equiv\left\{j \in \mathcal{N}_{i}-\mathcal{M}_{i}^{(l)} \mid r_{i j}<r_{\mathrm{c}}\right\}$.

Here, $\hat{E}(x)$ is the excess energy, which should be removed from the ordinary cutoff truncation sum $\sum_{j \in \mathcal{N}_{i}, r_{i j}<r_{\mathrm{c}}}$ for every $i$; and in this sense we call $\mathcal{J}_{i}^{(l)}$ an $e x$ cess subset, which generates non-zero multipoles inside a given cutoff sphere. From the above viewpoint, increasing the number of conditions, as in Eq. (5b), viz., increasing $l$, enables us to enhance the expression of the $L$ th ZM state $x$ in a given system, as long as $l \leq L$. This means that the expression of $\mathcal{M}_{i}^{(l)}$, and thus that of the excessive subset $\mathcal{J}_{i}^{(l)}$, are improved, and so the excessive energy becomes accurate. Thus, we expect that the energy value will become more accurate as $l(\leq L)$ increases. In fact, $\hat{E}(x)$ was approximated in the accuracy of $o\left(h^{0}\right)$ in the case of the monopole $(l=0)$ and of $o\left(h^{1}\right)$ in the case of the dipole $(l=1),{ }^{58}$ where $h$ is the displacement vector (see below for details). We keep in mind that the most appropriate moment $l$ should exist, depending on the system, except for unphysical or very high energy conditions. In fact, in our previous studies, for a sufficiently equilibrated, disordered system, or even an ordered crystal system, we found that $l=1$ describes the states better than $l=0$. Thus, a system that prefers a higher moment description should exist.

Along with the conditions (5), as well as in the dipole case, we employ the consistency condition:

$$
j \in \mathcal{J}_{i}^{(l)} \Leftrightarrow i \in \mathcal{J}_{j}^{(l)} \quad \text { for all } i, j(i \neq j) \in \mathcal{N} .
$$

This is required in order to subtract each excess interaction consistently; if we decide to remove the interaction with $j$ for $i$, viz., $j \in \mathcal{J}_{i}^{(l)}$, then we should also remove the interaction with $i$ for $j$, viz., $i \in \mathcal{J}_{j}^{(l)}$.

\section{Performing the scheme}

In order to conduct effective applications, we should construct an easily computable expression for estimating the excess energy, $\hat{E}(x)$, which is conceptually defined on the excess subset $\mathcal{J}_{i}^{(l)}$. To accomplish this, we approximate $\hat{E}(x)$ by a pairwise summation formula, by using conditions (5b) and (5c). Then, we can use a pairwise formula for both the second term on the right-hand side of Eq. (8) and the first term $\frac{1}{2} \sum_{i \in \mathcal{N}} \sum_{j \in \mathcal{R}_{i}} q_{i} q_{j} V\left(r_{i j}\right)$, where we often denote

$$
\begin{aligned}
& \mathcal{R}_{i} \equiv \mathcal{R}_{i}(x) \equiv\left\{j \in \mathcal{N}_{i} \mid r_{i j}<r_{\mathrm{c}}\right\}, \\
& \mathcal{R}^{i} \equiv \mathcal{R}^{i}(x) \equiv\left\{j \in \mathcal{N} \mid r_{i j}<r_{\mathrm{c}}\right\}
\end{aligned}
$$

to simply represent the summations.

We adopt two strategies, which can be summarized as follows:

I. Construct a certain polynomial to approximate $\hat{E}$ with respect to $\mathcal{J}_{i}^{(l)}$.

II. Represent the polynomial on $\mathcal{R}_{i}$.

\section{Strategy (I)}

a. Error description. The approximation of $\hat{E}$ is based on the fact that, from the (analytical) condition $(5 \mathrm{c}), \mathcal{J}_{i}^{(l)}$ is small in the sense that particles belonging in $\mathcal{J}_{i}^{(l)}$ are contained in a small area. Its smallness can be captured by the displacement from the position of the individual particle in $\mathcal{J}_{i}^{(l)}$ to the cutoff surface. As in Ref. 58, a displacement vector is introduced for any $x \in \Gamma$,

$$
h_{0}^{(i)} \equiv x-x^{(i)} \in \mathbb{R}^{d N},
$$

where

$$
\begin{aligned}
x^{(i)} & \equiv\left(x_{1}^{(i)}, \ldots, x_{N}^{(i)}\right), \\
x_{j}^{(i)} & \equiv\left\{\begin{array}{cc}
x_{i}+r_{\mathrm{c}} \frac{x_{j i}}{r_{j i}} & \text { if } j \in \mathcal{J}_{i}^{(l)} \\
x_{j} & \text { otherwise }
\end{array}\right\}
\end{aligned}
$$


for each $i \in \mathcal{N}$. Namely, $x_{j}^{(i)}$ for $j \in \mathcal{J}_{i}^{(l)}$ is the shifted position of $x_{j}$ obtained by expanding it along vector $x_{j i}$ to the cutoff surface; and for $j \notin \mathcal{J}_{i}^{(l)}$, we place $x_{j}^{(i)}$ at the original position, to maintain a consistent description (see Fig. 1). The displacement vector is small,

$$
h_{0}^{(i)} \equiv\left(h_{0,1}^{(i)}, \ldots, h_{0, N}^{(i)}\right) \sim 0 \in \mathbb{R}^{d N},
$$

by virtue of

$$
\left\|h_{0, j}^{(i)}\right\|=\left\{\begin{array}{cc}
r_{\mathrm{c}}-r_{i j} & \text { if } j \in \mathcal{J}_{i}^{(l)} \\
0 & \text { otherwise }
\end{array}\right\} \simeq 0
$$

Second, we suppose a motion (or perturbation) of the displacement vector, in order to specifically investigate the influence of the smallness of $\mathcal{J}_{i}^{(l)}$ on the degree of approximation for $\hat{E}$. This motion is basically done along the vector $h_{0}^{(i)}$, and we denote its domain by $I \equiv I_{i}^{x} \subset \mathbb{R}^{d N}$; according to the motion of $h^{(i)} \in I_{i}^{x}$, the positions $x^{(i)}+h^{(i)}$ vary. ${ }^{61}$

b. Approximation. For the approximation with respect to $\mathcal{J}_{i}^{(l)}$, we now directly use the fact that the particles in this subset are close to the cutoff surface. Considering the summation of $q_{j} V\left(r_{i j}\right)$ over $\mathcal{J}_{i}^{(l)}$, we approximate $V$ by a tractable polynomial near the cutoff distance. Specifically, we first approximate $V(r)$ by a series of even powers of $r$, in order to ensure a certain accuracy with respect to the displacement distance $r_{\mathrm{c}}-r$. That is, we construct an expansion satisfying

$$
V(r)=\sum_{m=0}^{l} a_{m}^{(l)} r^{2 m}+o\left(\left|r-r_{\mathrm{c}}\right|^{l}\right)\left(r \rightarrow r_{\mathrm{c}}\right) .
$$

When we fix the degree of the approximation with respect to $r_{\mathrm{c}}-r$ by $l$, and the degree of the polynomial by $2 l$ using the same number $l$, we have a unique answer for the values of real coefficients $\left\{a_{m}^{(l)}\right\}_{m=0,1, \ldots, l}$. We also see that the validity of Eq. (16) is equivalent to

$$
D^{m} V_{l}\left(r_{\mathrm{c}}\right)=D^{m} V\left(r_{\mathrm{c}}\right) \quad \text { for } m=0,1, \ldots, l,
$$

where

$$
V_{l}(r) \equiv \sum_{m=0}^{l} a_{m}^{(l)} r^{2 m}
$$

The details are described in Appendix A, and the results of the coefficients for several $l \mathrm{~s}$ are shown in Table I. The reason why we used only the even powers will be discussed in Sec. II D 2.
On the basis of these formulations, for each $i \in \mathcal{N}$, we see that the contribution of atom $i$ to the excess energy,

$$
\hat{E}_{i}(y) \equiv \hat{E}_{i}^{(l)}(y) \equiv \sum_{j \in \mathcal{J}_{i}^{(l)}} q_{i} q_{j} V\left(\left\|y_{i j}\right\|\right),
$$

for any $y \in \Gamma$ can be approximated by

$$
\breve{E}_{i}(y) \equiv \breve{E}_{i}^{(l)}(y) \equiv \sum_{j \in \mathcal{J}_{i}^{(l)}} q_{i} q_{j} V_{l}\left(\left\|y_{i j}\right\|\right)
$$

in the following sense: for any fixed $x \in \Gamma$ and $i \in \mathcal{N}$,

$$
\left(\hat{E}_{i}-\breve{E}_{i}\right)\left(x^{(i)}+h^{(i)}\right)=o\left(\left\|h^{(i)}\right\|^{l}\right)\left(I_{i}^{x} \ni h^{(i)} \rightarrow 0\right) .
$$

This can be proved using Eq. (16), as discussed in detailed in Appendix B.

Hence, for the excess energy [Eq. (9)], we see

$$
\hat{E}=\breve{E}+\Delta \quad\left(\text { or } \hat{E}^{(l)}=\breve{E}^{(l)}+\Delta^{(l)}\right),
$$

where

$$
\breve{E}=\frac{1}{2} \sum_{i \in \mathcal{N}} \breve{E}_{i}
$$

and

$$
\Delta \equiv \sum_{i \in \mathcal{N}} u_{i}
$$

with

$$
u_{i}\left(x^{(i)}+h^{(i)}\right)=o\left(\left\|h^{(i)}\right\|^{l}\right)\left(I_{i}^{x} \ni h^{(i)} \rightarrow 0\right)
$$

for any $x \in \Gamma$ and $i \in \mathcal{N}$. Since $\Delta$ is negligible, we have

$$
\hat{E} \simeq \breve{E} .
$$

In fact, for every $x \in \Gamma$ and $i \in \mathcal{N}$, from $x=x^{(i)}+h_{0}^{(i)}$ we

\begin{tabular}{|c|c|c|c|c|c|}
\hline$l$ & 0 & 1 & 2 & 3 & 4 \\
\hline$a_{0}^{(l)}$ & $b_{0}$ & $b_{0}-\frac{1}{2} b_{1} r_{c}$ & $\frac{1}{8} b_{2} r_{c}^{2}-\frac{5}{8} b_{1} r_{c}+b_{0}$ & $-\frac{1}{48} b_{3} r_{c}^{3}+\frac{3}{16} b_{2} r_{c}^{2}-\frac{11}{16} b_{1} r_{c}+b_{0}$ & $\frac{1}{384} b_{4} r_{c}^{4}-\frac{7}{192} b_{3} r_{c}^{3}+\frac{29}{128} b_{2} r_{c}^{2}-\frac{93}{128} b_{1} r_{c}+b_{0}$ \\
\hline$a_{1}^{(l)}$ & & $\frac{1}{2} \frac{b_{1}}{r_{c}}$ & $\frac{3}{4} \frac{b_{1}}{r_{c}}-\frac{1}{4} b_{2}$ & $\frac{15}{16} \frac{b_{1}}{r_{c}}-\frac{7}{16} b_{2}+\frac{1}{16} b_{3} r_{c}$ & $\frac{35}{32} \frac{b_{1}}{r_{c}}-\frac{19}{32} b_{2}-\frac{1}{96} b_{4} r_{c}^{2}+\frac{1}{8} b_{3} r_{c}$ \\
\hline$a_{2}^{(l)}$ & & & $\frac{1}{8} \frac{b_{2}}{r_{c}^{2}}-\frac{1}{8} \frac{b_{1}}{r_{c}^{3}}$ & $\frac{5}{16} \frac{b_{2}}{r_{c}^{2}}-\frac{5}{16} \frac{b_{1}}{r_{c}^{3}}-\frac{1}{16} \frac{b_{3}}{r_{c}}$ & $\frac{1}{64} b_{4}-\frac{35}{64} \frac{b_{1}}{r_{c}^{3}}+\frac{35}{64} \frac{b_{2}}{r_{c}^{2}}-\frac{5}{32} \frac{b_{3}}{r_{c}}$ \\
\hline$a_{3}^{(l)}$ & & & & $\frac{1}{16} \frac{b_{1}}{r_{c}^{5}}-\frac{1}{16} \frac{b_{2}}{r_{c}^{4}}+\frac{1}{48} \frac{b_{3}}{r_{c}^{3}}$ & $\frac{7}{32} \frac{b_{1}}{r_{c}^{5}}-\frac{7}{32} \frac{b_{2}}{r_{c}^{4}}+\frac{1}{12} \frac{b_{3}}{r_{c}^{3}}-\frac{1}{96} \frac{b_{4}}{r_{c}^{2}}$ \\
\hline$a_{4}^{(l)}$ & & & & & $\frac{5}{128} \frac{b_{2}}{r_{c}^{6}}-\frac{5}{128} \frac{b_{1}}{r_{c}^{7}}-\frac{1}{64} \frac{b_{3}}{r_{c}^{5}}+\frac{1}{384} \frac{b_{4}}{r_{c}^{4}}$ \\
\hline
\end{tabular}
have $u_{i}(x)=u_{i}\left(x^{(i)}+h_{0}^{(i)}\right)$, which is $\simeq 0$, by virtue of the behavior described in Eq. (25) and the small size of the displacement vector $h_{0}^{(i)} \in I_{i}^{x}$ [Eq. (14)]. Note that better accuracy of the excess energy value in the ZM state can be attained with a larger $l$.

\section{Strategy (II)}

The remaining task is to represent Eq. (20) on $\mathcal{R}_{i}$, by converting the sum into a usual pairwise cutoff form. By intensive use of the neutrality condition, Eq. (5b), as well as the consistency condition (10), we can evaluate the sum $\sum_{i \in \mathcal{N}} \sum_{j \in \mathcal{J}_{i}^{(l)}} q_{i} q_{j} r_{i j}^{2 m}$ in a simple manner. Specifically,

TABLE I. Coefficients in Eq. (16) [the solution of Eq. (17)]. Here, $b_{n} \equiv D^{n} V\left(r_{\mathrm{c}}\right)$ is the $n$th derivative of $V$ at $r_{\mathrm{c}}(n=0,1, \ldots, l)$. 
we show

$$
\begin{aligned}
& \sum_{i \in \mathcal{N}} \sum_{j \in \mathcal{J}_{i}^{(l)}} q_{i} q_{j} r_{i j}^{2 m} \\
= & \sum_{i \in \mathcal{N}} \sum_{j \in \mathcal{R}^{i}} q_{i} q_{j} r_{i j}^{2 m} .
\end{aligned}
$$

Aside from the details of the proof of Eq. (27), which is completed in Appendix C, here we note that the validity of this equivalence is due to the fact that the even power of $r_{i j}$ enables the purely algebraic expansion of $r_{i j}^{n}=\left[\left\|x_{i}\right\|^{2}\right.$ $\left.+\left\|x_{j}\right\|^{2}-2\left(x_{i} \mid x_{j}\right)\right]^{n / 2}$. In contrast, an odd power of $r_{i j}$ prevents such an expansion, and so does the inclusion of an odd power in the polynomial, Eq. (18). Namely, although we can define any type of expansion in Eq. (16) in principle, we find that a polynomial with only even powers has a high affinity with the algebraic condition, Eq. (5b).

Now, we have an approximation with a specifically convenient form, viz.; the approximate excess energy, Eq. (23), becomes

$$
\begin{aligned}
\breve{E}(x)= & \frac{1}{2} \sum_{i \in \mathcal{N}} \sum_{j \in \mathcal{J}_{i}^{(l)}} q_{i} q_{j} V_{l}\left(r_{i j}\right) \\
= & \frac{1}{2} \sum_{m=0}^{l} a_{m}^{(l)} \sum_{i \in \mathcal{N}} \sum_{j \in \mathcal{J}_{i}^{(l)}} q_{i} q_{j} r_{i j}^{2 m} \\
= & \frac{1}{2} \sum_{m=0}^{l} a_{m}^{(l)} \sum_{i \in \mathcal{N}} \sum_{j \in \mathcal{R}^{i}} q_{i} q_{j} r_{i j}^{2 m} \\
= & \frac{1}{2} \sum_{i \in \mathcal{N}} \sum_{j \in \mathcal{R}_{i}} \sum_{m=0}^{l} q_{i} q_{j} a_{m}^{(l)} r_{i j}^{2 m} \\
& +\frac{1}{2} \sum_{i \in \mathcal{N}} q_{i}^{2} a_{0}^{(l)} .
\end{aligned}
$$

In Eq. (28d), we used $\mathcal{R}_{i}$, instead of $\mathcal{R}^{i}$, and the fact that $\sum_{m=0}^{l} a_{m}^{(l)} r_{i j}^{2 m}=a_{0}^{(l)}$ for $i=j$.

\section{Summary of formula}

Therefore, from Eqs. (22) and (28), we obtain

$$
\hat{E}=\check{E}+\kappa+\Delta \text {. }
$$

Here,

$$
\begin{aligned}
\check{E}(x) & \equiv \frac{1}{2} \sum_{i \in \mathcal{N}} \sum_{j \in \mathcal{R}_{i}} \sum_{m=0}^{l} q_{i} q_{j} a_{m}^{(l)} r_{i j}^{2 m} \\
& =\frac{1}{2} \sum_{i \in \mathcal{N}} \sum_{j \in \mathcal{R}_{i}} q_{i} q_{j} V_{l}\left(r_{i j}\right)
\end{aligned}
$$

adopts a pairwise expression,

$$
\kappa \equiv \kappa^{(l)} \equiv \frac{1}{2} a_{0}^{(l)} \sum_{i \in \mathcal{N}} q_{i}^{2}
$$

forms a constant, and $\Delta$ is the negligible part, as demonstrated. Combining Eqs. (29)-(31) with Eq. (8), we conclude that an approximation to the total energy, for $x \in \Gamma$, is given by the ZM energy, viz.,

$$
E(x) \simeq E_{\mathrm{ZM}}^{(l)}(x)
$$

with

$$
\begin{aligned}
E_{\mathrm{ZM}}^{(l)}(x) \equiv & \frac{1}{2} \sum_{i \in \mathcal{N}} \sum_{\substack{j \in \mathcal{N}_{i} \\
r_{i j}<r_{\mathrm{c}}}} q_{i} q_{j}\left[V\left(r_{i j}\right)-\sum_{m=0}^{l} a_{m}^{(l)} r_{i j}^{2 m}\right] \\
& -\left(\frac{a_{0}^{(l)}}{2}+\frac{\alpha}{\sqrt{\pi}}\right) \sum_{i \in \mathcal{N}} q_{i}^{2} .
\end{aligned}
$$

The coefficients $\left\{a_{m}^{(l)}\right\}_{m=0,1, \ldots, l}$ (Table I) are defined by $r_{\mathrm{c}}$ and higher derivatives of $V$ at $r_{\mathrm{c}}$. They are thus completely determined by $r_{\mathrm{c}}$ and $\alpha$, and can be evaluated in advance of the simulations. If we use $\alpha=0$ (no damping case), then Eq. (33) reduces to Eq. (1), with coefficients with different values from those in the $\alpha>0$ case.

The force acting on each atom $i$ is thus consistently derived, as

$$
\begin{aligned}
F_{i}^{(l)}(x) & \equiv-\nabla_{i} E_{\mathrm{ZM}}^{(l)}(x) \\
& =\sum_{\substack{j \in \mathcal{N}_{i} \\
r_{i j}<r_{\mathrm{c}}}} q_{i} q_{j} e^{(l)}\left(r_{i j}\right) \frac{x_{i j}}{r_{i j}} \in \mathbb{R}^{d},
\end{aligned}
$$

for any $x \in \Gamma$ and for $l \geq 1$, where

$$
e^{(l)}(r) \equiv F(r)+\sum_{m=1}^{l} 2 m a_{m}^{(l)} r^{2 m-1}
$$

with

$$
F(r) \equiv-D V(r)=\frac{\operatorname{erfc}(\alpha r)}{r^{2}}+\frac{2 \alpha}{\sqrt{\pi}} \frac{\exp \left(-\alpha^{2} r^{2}\right)}{r} .
$$

\section{RESULTS AND DISCUSSION}

\section{A. Energy functional form}

Note that the ZM energy $E_{\mathrm{ZM}}^{(l)}(x)$ can also be represented in a simple and "unified" form ${ }^{14}$ as

$$
\begin{aligned}
E_{\mathrm{ZM}}^{(l)}(x)= & \frac{1}{2} \sum_{i \in \mathcal{N}} \sum_{\substack{j \in \mathcal{N}_{i} \\
r_{i j}<r_{\mathrm{c}}}} q_{i} q_{j}\left[u^{(l)}\left(r_{i j}\right)-u^{(l)}\left(r_{\mathrm{c}}\right)\right] \\
& -\frac{1}{2}\left[u^{(l)}\left(r_{\mathrm{c}}\right)+\frac{2 \alpha}{\sqrt{\pi}}\right] \sum_{i \in \mathcal{N}} q_{i}^{2},
\end{aligned}
$$

employing the function

$$
u^{(l)}(r) \equiv\left\{\begin{array}{cc}
V(r)-\sum_{m=1}^{l} a_{m}^{(l)} r^{2 m} & \text { if } l \geq 1 \\
V(r) & \text { if } l=0
\end{array}\right\},
$$

in which the polynomial in Eq. (38) omits the $m=0$ term in Eqs. (18) and (33). Equation (37) can be obtained using the fact that $V\left(r_{\mathrm{c}}\right)=V_{l}\left(r_{\mathrm{c}}\right)$, and so $a_{0}^{(l)}=u^{(l)}\left(r_{\mathrm{c}}\right)$; see Eq. (17).

Furthermore, this pair potential function in Eq. (37),

$$
\mathcal{U}^{(l)} \equiv u^{(l)}-u^{(l)}\left(r_{\mathrm{c}}\right)=V-V_{l},
$$




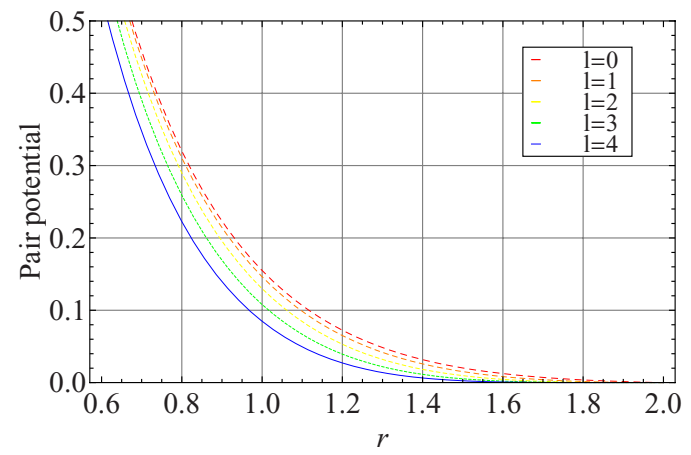

FIG. 2. Pair potential functions in the ZM method with moment $l=0,1$, $2,3,4$, from the top to bottom, respectively. A normalized unit, using a fundamental length, is applied for the cutoff distance, $r_{\mathrm{c}}=2$ [Length], and the damping parameter, $\alpha=1\left[\right.$ Length $\left.^{-1}\right]$.

dampens to zero at $r=r_{\mathrm{c}}$ smoothly with increase in the moment $l$, as seen

$$
\begin{aligned}
D^{m} \mathcal{U}^{(l)}\left(r_{\mathrm{c}}\right) & =D^{m} V\left(r_{\mathrm{c}}\right)-D^{m} V_{l}\left(r_{\mathrm{c}}\right) \\
& =0 \quad \text { for } m=0, \ldots, l
\end{aligned}
$$

Namely, the function

$$
(0, \infty) \ni r \mapsto\left\{\begin{array}{cl}
\mathcal{U}^{(l)}(r) & \text { for } r<r_{\mathrm{c}} \\
0 & \text { for } r \geq r_{\mathrm{c}}
\end{array}\right\}
$$

has a smoothness of class $C^{l}(l \geq 0)$. In particular, the smoothness for $m=0,1 \leq l$ leads to the consistent force function given in Eq. (34) [note that the $l=0$ case, i.e., the ZC case, is exceptional regarding the force, since Eq. (41) only gives the continuity ${ }^{56}$ ]. Figure 2 shows the curve of the pair potential $\mathcal{U}^{(l)}$, indicating the damping with increasing values of the moment $l$.

\section{B. Error analysis}

The excess energy approximation is more accurate as $l$ becomes larger in the $L$ th ZM state with $l \leq L$. The approximation for $l=0$ is represented as, for any $x \in \Gamma$ and $i \in \mathcal{N}$,

$$
\left(\hat{E}_{i}^{(0)}-\breve{E}_{i}^{(0)}\right)\left(x^{(i)}+h^{(i)}\right)=o(1)\left(I_{i}^{x} \ni h^{(i)} \rightarrow 0\right),
$$

and that for $l=1$ is

$$
\left(\hat{E}_{i}^{(1)}-\breve{E}_{i}^{(1)}\right)\left(x^{(i)}+h^{(i)}\right)=o\left(\left\|h^{(i)}\right\|\right)\left(I_{i}^{x} \ni h^{(i)} \rightarrow 0\right) .
$$

As described in Sec. III C, the ZM method with $l=0$ is equal to the ZC scheme, and that with $l=1$ is equal to the $\mathrm{ZD}$ scheme. In fact, these errors are shown in Ref. 58 as those of the ZC and ZD schemes, respectively. By the currently derived $\mathrm{ZM}$ scheme, for $l \geq 2$, we obtain a higher accuracy,

$$
\left(\hat{E}_{i}^{(l)}-\breve{E}_{i}^{(l)}\right)\left(x^{(i)}+h^{(i)}\right)=o\left(\left\|h^{(i)}\right\|^{l}\right)\left(I_{i}^{x} \ni h^{(i)} \rightarrow 0\right) .
$$

In contrast, the above approximating features in the $\mathrm{ZC}$ and ZD schemes are also helpful to explain the degree of another approximation in the ZM scheme, i.e., Eq. (7), which concerns the replacement strategy. That is, regarding the ZM state $x \in \Gamma=\Gamma_{l}$ for $l>1$, we obtain the following relations:

$$
\begin{gathered}
\frac{1}{2} \sum_{i \in \mathcal{N}} \sum_{j \in \mathcal{N}_{i}} q_{i} q_{j} V\left(r_{i j}\right) \\
\simeq \frac{1}{2} \sum_{i \in \mathcal{N}} \sum_{j \in \mathcal{N}_{i},} q_{i} q_{j}\left[u^{(m)}\left(r_{i j}\right)-u^{(m)}\left(r_{\mathrm{c}}\right)\right] \\
-\frac{u^{(m)}\left(r_{\mathrm{c}}\right)}{2} \sum_{i \in \mathcal{N}} q_{i}^{2}(m=0 \text { or } 1) \\
\simeq \frac{1}{2} \sum_{i \in \mathcal{N}} \sum_{j \in \mathcal{M}_{i}^{(l)}} q_{i} q_{j} V\left(r_{i j}\right) .
\end{gathered}
$$

The near equality in Eq. (46) is indicated by the fact that the ZC or ZD energy works well for $x \in \Gamma_{0}$ or $x \in \Gamma_{1}$, respectively, as shown in many works, such as those discussed in Sec. I. Although these results were obtained by adding the approximation represented by Eq. (4), this approximation is common for all $l$. In addition, although these results have been justified regarding the states $x \in \Gamma_{0}$ or $\Gamma_{1}$, we note that this near equality is passed on to $x \in \Gamma_{l}$, since $\Gamma_{0} \supset \Gamma_{1} \supset \cdots \supset \Gamma_{l}$ holds. The next near equality in Eq. (47) is due to the fact that we can restrict condition (5b) to only $m=0$ or $m=1$ even if $l>1$, thus obtaining the ZC or ZD scheme, respectively. Therefore, the above relation is one of the quantitative justifications of Eq. (7) for $l>1$. The justifications for $l \leq 1$ have been discussed previously. ${ }^{56,58}$

To reiterate, the $\mathrm{ZM}$ method provides the uniqueness of the coefficients of the polynomial used in it. Namely, if we fix the value of the moment $l$, which is also the degree of the accuracy of the excess energy and half of the degree of the polynomial with even powers for approximating the potential function $V$ on the excess subset, then the set of the coefficients of the polynomial, viz., the polynomial itself, is uniquely determined. In this sense, for example, when we fix $l=1$, the functional form used in the ZD method [see Eq. (49) below] is derived uniquely. This fact was not clarified in Ref. 58, and is revealed here for the first time.

\section{Relationship with other methods}

For $l=0$ in the current method, from the definition of $u^{(0)}$ [Eq. (38)], we see

$$
\begin{aligned}
E_{\mathrm{ZM}}^{(0)}(x)= & \frac{1}{2} \sum_{i \in \mathcal{N}} \sum_{\substack{j \in \mathcal{N}_{i} \\
r_{i j}<r_{\mathrm{c}}}} q_{i} q_{j}\left[V\left(r_{i j}\right)-V\left(r_{\mathrm{c}}\right)\right] \\
& -\frac{1}{2}\left[V\left(r_{\mathrm{c}}\right)+\frac{2 \alpha}{\sqrt{\pi}}\right] \sum_{i \in \mathcal{N}} q_{i}^{2} .
\end{aligned}
$$

Namely, the ZM energy $E_{\mathrm{ZM}}^{(0)}(x)$ is the energy by Wolf et $a l .{ }^{33}$ In the case of $l=1$, as seen from Table I, we have 


$$
\begin{gathered}
u^{(1)}(r)=V(r)-a_{1}^{(1)} r^{2}=V(r)-\frac{D V\left(r_{\mathrm{c}}\right)}{2 r_{c}} r^{2} \equiv u_{\mathrm{ZD}}(r), \text { and } \\
E_{\mathrm{ZM}}^{(1)}(x)=\frac{1}{2} \sum_{i \in \mathcal{N}} \sum_{\substack{j \in \mathcal{N}_{i} \\
r_{i j}<r_{\mathrm{c}}}} q_{i} q_{j}\left[u_{\mathrm{ZD}}\left(r_{i j}\right)-u_{\mathrm{ZD}}\left(r_{\mathrm{c}}\right)\right] \\
\\
-\frac{1}{2}\left[u_{\mathrm{ZD}}\left(r_{\mathrm{c}}\right)+\frac{2 \alpha}{\sqrt{\pi}}\right] \sum_{i \in \mathcal{N}} q_{i}^{2}
\end{gathered}
$$

gives the ZD energy. ${ }^{58}$ Thus, the ZM method is a generalization of the Wolf and ZD methods, which are included as special cases of the ZM method with $l=0$ and 1 , respectively.

Two other schemes regarding $l=0$ are discussed. First, to consistently conduct the MD simulation [by recovering sufficient smoothness of the pair function (Sec. III A)], another type of ZC scheme, the force-switching Wolf method, ${ }^{56}$ was derived and takes the form of

$$
\begin{aligned}
E_{\mathrm{FSW}}(x) \equiv & \frac{1}{2} \sum_{i \in \mathcal{N}} \sum_{\substack{j \in \mathcal{N}_{i} \\
r_{i j}<r_{\mathrm{c}}}} q_{i} q_{j}\left[\hat{V}\left(r_{i j}\right)-\hat{V}\left(r_{\mathrm{c}}\right)\right] \\
& -\frac{1}{2}\left[\hat{V}\left(r_{\mathrm{c}}\right)+\frac{2 \alpha}{\sqrt{\pi}}\right] \sum_{i \in \mathcal{N}} q_{i}^{2},
\end{aligned}
$$

with

$$
\hat{V}(r) \equiv\left\{\begin{array}{cl}
V(r) & \text { for } 0<r<r_{1} \\
V^{*}(r)+V\left(r_{1}\right)-V^{*}\left(r_{1}\right) & \text { for } r_{1} \leq r \leq r_{\mathrm{c}} \\
V\left(r_{1}\right)-V^{*}\left(r_{1}\right) & \text { for } r_{\mathrm{c}}<r<\infty
\end{array}\right.
$$

Here, $V^{*}$ is a switching function with $V^{*}\left(r_{\mathrm{c}}\right)=0$ and $r_{1}$ is the switching length, for which the limit $r_{1} \rightarrow r_{\mathrm{c}}$ gives the Wolf method or the ZM method with $l=0$. Second, the ZM method with $l=0$ and $\alpha=0$, viz., the non-damped Wolf method, can be considered to be the same as the scheme proposed by Harrison $^{62}$ when calculating the Madelung energy for a simple structure. This can be seen by noting that the energy with respect to atom $i$ in Eq. (48) with $\alpha=0$ is

$$
\begin{aligned}
E_{\mathrm{ZM}, i}^{(0)}(x) & =\sum_{\substack{j \in \mathcal{N}_{i} \\
r_{i j}<r_{\mathrm{c}}}} q_{i} q_{j}\left[1 / r_{i j}-1 / r_{\mathrm{c}}\right]-\left(1 / r_{\mathrm{c}}\right) q_{i}^{2} \\
& =q_{i}\left[\sum_{\substack{j \in \mathcal{N}_{i} \\
r_{i j}<r_{\mathrm{c}}}} q_{j} / r_{i j}-Q_{i} / r_{\mathrm{c}}\right]
\end{aligned}
$$

where $Q_{i} \equiv \sum_{j \in \mathcal{N}, r_{i j}<r_{\mathrm{c}}} q_{j}$ is the net charge in the cutoff sphere of radius $r_{\mathrm{c}},{ }^{14,33}$ and yields the correction. ${ }^{62}$

Furthermore, Eq. (37) adopts a unified form utilized in non-Ewald methods. ${ }^{14}$ Among them, the averaging notion is useful, as shown by the pre-averaging (PA) procedure introduced by Yakub and Ronchi. ${ }^{21}$ The energy formula is derived by averaging the quantities over the spherical angular coordinates in the expansion of the Ewald method. The energy formula can be written as

$$
\begin{aligned}
E_{\mathrm{PA}}(x)= & \frac{1}{2} \sum_{i \in \mathcal{N}} \sum_{\substack{j \in \mathcal{N}_{i} \\
r_{i j}<r_{\mathrm{m}}}} q_{i} q_{j}\left[V_{\mathrm{PA}}\left(r_{i j}\right)-V_{\mathrm{PA}}\left(r_{\mathrm{m}}\right)\right] \\
& -\frac{1}{2} V_{\mathrm{PA}}\left(r_{\mathrm{m}}\right) \sum_{i \in \mathcal{N}} q_{i}^{2}
\end{aligned}
$$

with

$$
V_{\mathrm{PA}}(r) \equiv \frac{1}{r}\left\{1+\frac{1}{2}\left(\frac{r}{r_{\mathrm{m}}}\right)^{3}\right\},
$$

and $r_{\mathrm{m}}$ being the radius of the volume-equivalent sphere of the MD cubic cell with edge $L$, defined by $(4 / 3) \pi r_{\mathrm{m}}^{3}=L^{3}$. The energy, Eq. (53), is also obtained if we take the limits of $r_{\mathrm{c}} \rightarrow r_{\mathrm{m}}$ and $\alpha \rightarrow 0$ in the ZD method, viz., the ZM method with $l=1$, noting that the $2 \alpha / \sqrt{\pi}$ term eventually vanishes with $\alpha=0$. A similar relationship is also found in the RF method. Its energy formula ${ }^{63,64}$ in a slightly modified form ${ }^{14}$ can be represented by

$$
\begin{aligned}
E_{\mathrm{MRF}}(x)= & \frac{1}{2} \sum_{i \in \mathcal{N}} \sum_{\substack{j \in \mathcal{N}_{i} \\
r_{i j}<r_{\mathrm{c}}}} q_{i} q_{j}\left[V_{\mathrm{RF}}\left(r_{i j}\right)-V_{\mathrm{RF}}\left(r_{\mathrm{c}}\right)\right] \\
& -\frac{1}{2} V_{\mathrm{RF}}\left(r_{\mathrm{c}}\right) \sum_{i \in \mathcal{N}} q_{i}^{2},
\end{aligned}
$$

where

$$
V_{\mathrm{RF}}(r) \equiv \frac{1}{r}\left\{1+\frac{\epsilon_{\mathrm{RF}}-1}{2 \epsilon_{\mathrm{RF}}+1}\left(\frac{r}{r_{\mathrm{c}}}\right)^{3}\right\} .
$$

The limit to infinity of the dielectric constant of the environment outside the cutoff sphere, i.e., $\epsilon_{\mathrm{RF}} \rightarrow \infty$, provides the ZD summation energy (49) with $\alpha=0$.

These relationships among the individual methods are summarized in Fig. 3, and suggest that Eq. (37) is in fact a "unified" form. These last two methods seem to be irrelevant to the charge neutralization idea, at least in their original derivations. Deep insight would reveal the physical basis of these nontrivial relationships. ${ }^{14,58,65}$

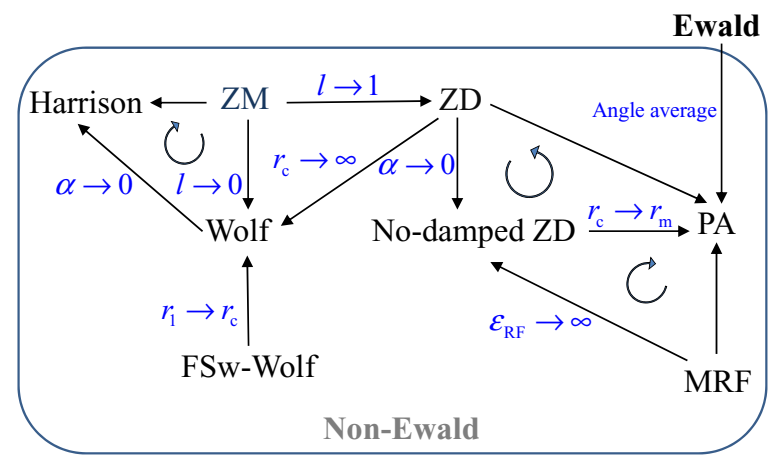

FIG. 3. Schematic relationships among the methods for calculating electrostatic interactions. The shaded region shows the intimately related nonEwald methods, including the zero-multipole (ZM) method, zero-dipole (ZD) method, Wolf method, Harrison method, force-switching Wolf (FSw-Wolf) method, and pre-averaging (PA) method. No-damped ZD and MRF indicate the ZD method with $\alpha=0$ and the modified reaction field method represented by Eq. (55), respectively. The round arrow shows the commutativity among the three relationships. 
Along the lines of the neutralization, Abarenkov ${ }^{66}$ proposed a different idea, with the help of the regrouping method.$^{67}$ It is the interesting notion of the lattice potential unit cell, which is a generalized unit cell, and the atomic energy $E_{i}(x)$ is efficiently evaluated, under the PBC. This new unit cell contains additional particles located at certain lattice points. The charge values of these particles are sought to neutralize the multipole moments of the cell up to an arbitrary higher order, and their Ewald potential contributions are totally zero. Although we do not currently know the direct relationship between this method and the ZM method, reviving the view of the image-charges,${ }^{58}$ which are placed on the cutoff sphere, in the latter method might be useful to consider the relationship.

\section{Numerical considerations}

To illustrate the numerical efficiency of the current method, we will first examine its application to an $\mathrm{NaCl}$ crystal system, which is simple and prototypic. An fcc cell with the normalized unit-cell edge length and the normalized charge values was used under the 3-dimensional periodic boundary conditions, and Madelung constant was estimated. The evaluation of Madelung constants is a classical solid-state problem, and a simple estimation is useful for solving physical problems, ${ }^{62}$ including studies of charge transfer in metallic alloys ${ }^{68}$ and of a valence-skipping compound. ${ }^{69}$ On the other hand, its rigorous estimation is related to certain mathematical objects such as zeta functions ${ }^{70}$ which are relevant to the pursuit of the mathematical definition of the constant, owing to the conditionally convergent feature.

Here, the energy corresponding to particle $i$ was considered to be given, from Eq. (37), as

$$
\begin{aligned}
E_{\mathrm{ZM}, i}^{(l)}(x) \equiv & \sum_{\substack{j \in \mathcal{N}_{i} \\
r_{i j}<r_{\mathrm{c}}}} q_{i} q_{j}\left[u^{(l)}\left(r_{i j}\right)-u^{(l)}\left(r_{\mathrm{c}}\right)\right] \\
& -\left[u^{(l)}\left(r_{\mathrm{c}}\right)+\frac{2 \alpha}{\sqrt{\pi}}\right] q_{i}^{2},
\end{aligned}
$$

and Madelung constant was estimated from $M^{(l)} \equiv-R_{\mathrm{m}}$. $E_{\mathrm{ZM}, i}^{(l)}\left(x_{\mathrm{C}}\right)$, with the nearest-neighbor ionic distance $R_{\mathrm{m}}$ $=1 / 2$, the crystal state $x_{\mathrm{C}}$, and the use of $i=1$. In this system, $M^{(l)}$ is irrelevant to the choice of $i$. The error of the constant obtained by the ZM method with moment $l$ was estimated by $\left(M^{(l)}-M_{\text {Theo }}\right) / M_{\text {Theo }}$, where $M_{\text {Theo }}$ $\equiv 1.74756459463318$ was used. ${ }^{70}$

The results are shown in Fig. 4. The errors show convergent behaviors with increasing values of the cutoff length $r_{\mathrm{c}}$. With increases in the moment $l$, which facilitates the higher order multipole-moment neutralization, the accuracy becomes higher on average, and faster convergent behavior is observed. In addition, the error variations with increasing $r_{\mathrm{c}}$ become smoother. For example, the error was -0.0014 at only $r_{\mathrm{c}}=1.5$ for $l=4$. The current method includes the damping parameter $\alpha$ (with the physical dimension of the inverse of length), and the value used in Fig. 4 was $\alpha=1$.

A more suitable value of $\alpha$ provided better efficiency. Figure 5 shows the absolute value of the error for the scheme with $l=4$, using several values of $\alpha$. First of all, an accuracy

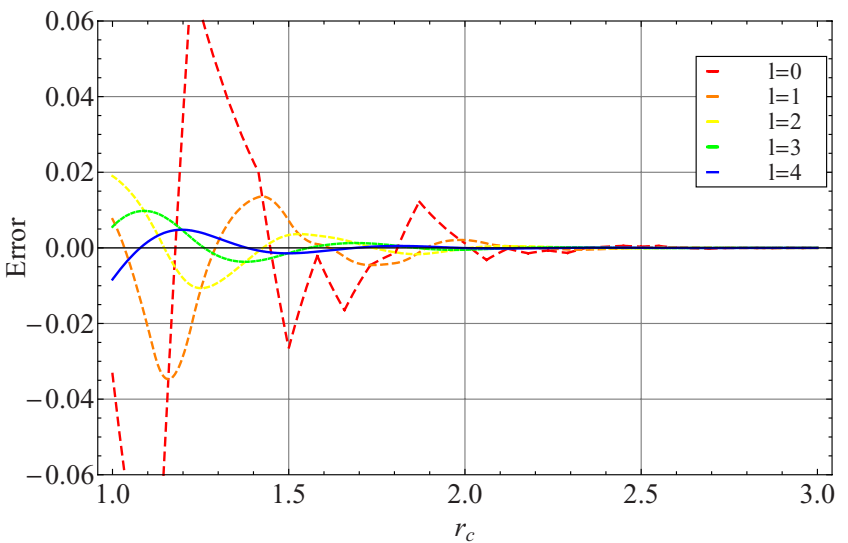

FIG. 4. The error of Madelung constant of the $\mathrm{NaCl}$ crystal with respect to the cutoff length $r_{\mathrm{c}}$. A normalized length was used such that the nearestneighbor ionic distance was $1 / 2$ (unit-cell edge length was set to 1). Madelung constant was estimated by the ZM method with $\alpha=1$, using individual values of the moment, $l=0,1,2,3,4$.

on the order of $10^{-6}$ can be attained at $r_{\mathrm{c}} \sim 2$. For example, the error was $7.8 \times 10^{-6}$ for $\alpha=1.5$ and $1.3 \times 10^{-6}$ for $\alpha=1.3$, both at $r_{\mathrm{c}}=2$. By increasing the cutoff distance to $r_{\mathrm{c}} \sim 5$, we achieved an accuracy of about 13 decimal places; e.g., the error was $-7.5 \times 10^{-13}$ for $r_{\mathrm{c}}=5$ and $\alpha=1$.

Since it is known that a simple cubic (not spherical) truncation using the minimum image convention (MIC) may produce results as good as the Ewald sums for low electrostatic coupling ${ }^{71}$, Madelung constant was also calculated by the cubic MIC, as shown in Fig. 5. The errors by the MIC calculation were not bad, e.g., the error was $-3.0 \times 10^{-4}$ at $r_{\mathrm{c}}=3$, where $r_{\mathrm{c}}$ refers to the number of unit cells along one axis (unit-cell edge length was 1). However, the convergence was slower, as compared with the results of the $\mathrm{ZM}$ method. The error of the cubic MIC at $r_{\mathrm{c}} \sim 6$ can be attained by the ZM method at $r_{\mathrm{c}} \sim 2$.

Instead of the normalized length we used, when we consider a physical unit of length, such as a lattice constant of $R$ $\sim 5.6 \AA$, the normalized range $2 \lesssim r_{\mathrm{c}} \lesssim 3$ corresponds to a

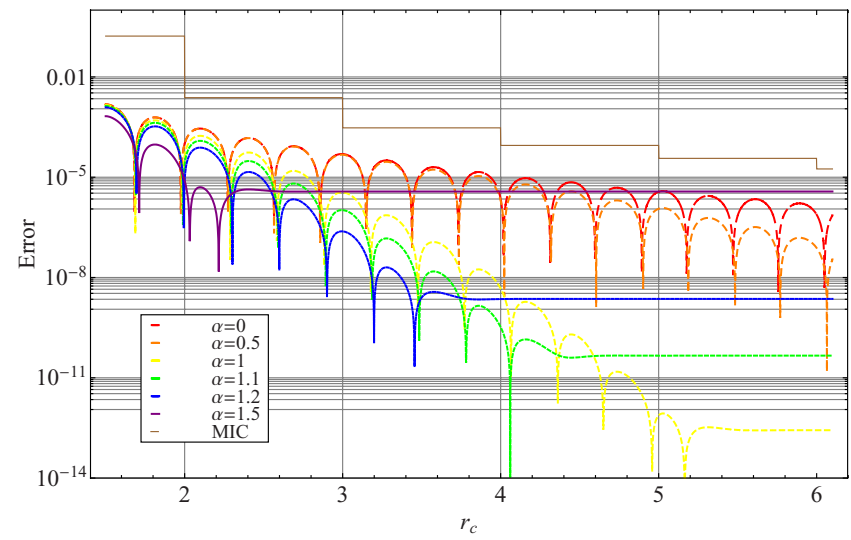

FIG. 5. The absolute value of the error (log scale) of Madelung constant of the $\mathrm{NaCl}$ crystal, obtained by the $\mathrm{ZM}$ method with moment $l=4$. Several values of the damping parameter $\alpha$ [Length ${ }^{-1}$ ] were used. The cutoff length $r_{\mathrm{c}}$ was varied from 1.5 to 6 , and the nearest-neighbor ionic distance was set to $1 / 2$. For comparison, the results obtained by the cubic MIC method is shown by the step function, which is defined as $\Delta_{\text {MIC }}\left(r_{\mathrm{c}}\right)=\Delta_{n}$ for $n \leq r_{\mathrm{c}}<n+1$ $(n=1, \ldots, 6)$, where $\Delta_{n}$ is the absolute value of the error when the MIC utilizes $n$ unit-cells along one axis. 


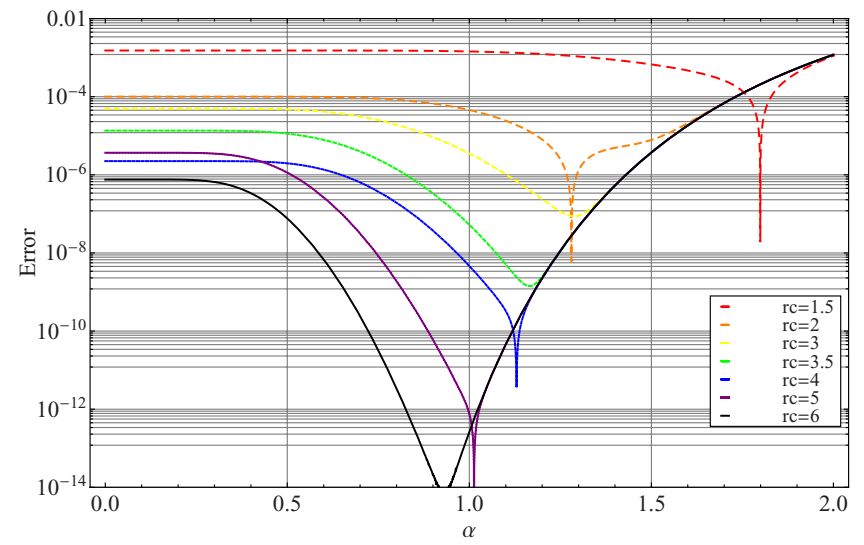

FIG. 6. The absolute value of the error (log scale) of Madelung constant of the $\mathrm{NaCl}$ crystal, obtained by the $\mathrm{ZM}$ method with moment $l=4$. The dependence on $\alpha$ [Length ${ }^{-1}$ ] is explicitly shown for the indicated fixed values of the cutoff length $r_{\mathrm{c}}$ (nearest-neighbor ionic distance was 1/2).

physical range $11 \AA 2 r_{\mathrm{c}} \lesssim 16 \AA$, which is a practical cutoff distance region in molecular simulations. In this range, the optimum $\alpha$ value of the ZM method was near 1.3, as seen in Fig. 6, where the absolute value of the error was plotted against $\alpha$, with distinct $r_{\mathrm{c}}$ values. In fact, the error was 9.0 $\times 10^{-8}$ for $\alpha=1.3$ and $r_{\mathrm{c}}=3$. This value, $\alpha=1.3$, corresponding to $1.3 / R \sim 0.23 \AA^{-1}$ in a real physical unit, is nearly in accord with the optimum values shown in previous studies, such as those for the $\mathrm{ZC}^{34}$ and $\mathrm{ZD}^{58}$ schemes.

If impressively high accuracy is not required, then fine optimization of $\alpha$ is not necessarily needed. In fact, as shown in Fig. 6, the dependence on $\alpha$ is not impractical, and the cusp simply indicates the reverse of the signature of the error. If we satisfy the accuracy within the order of $10^{-5}$ at $r_{\mathrm{c}} \gtrsim 3$, then $0 \leq \alpha \lesssim 1.5$ gives the error within it.

The optimization of $\alpha$ would be needed when we require a faster result (viz., the same accuracy with a shorter $r_{\mathrm{c}}$ ) or a smaller error. As shown in Figs. 5 and 6, a faster result can be obtained by setting a larger $\alpha$ in a suitable range. This is the advantage of a larger $\alpha$. However, the disadvantage of a large $\alpha$ is the error saturation; e.g., the error for $\alpha=1.5$ was not appreciably reduced regardless of increasing $r_{\mathrm{c}}$ over 2.5 (Fig. 5). This is the consequence of the error represented in Eq. (4). In contrast, regarding a smaller $\alpha$, we face a slower result but can retard the saturation. Figure 6 also suggests that a smaller $\alpha$ is suited to a larger $r_{\mathrm{c}}$. The limit case is $\alpha=0$ (see also the discussion in Ref. 72), which should behave nicely for longer $r_{\mathrm{c}}$, and this conforms to the idea proposed by Harrison, ${ }^{62}$ utilizing no damping.

As our second example, Madelung constant of another prototypic crystal system, a $\mathrm{CsCl}$ crystal with a simple cubic structure, was examined in a similar manner to the $\mathrm{NaCl}$ case. The constants ${ }^{73}$ used were $R_{\mathrm{m}}=\sqrt{3} / 2$ and $M_{\text {Theo }}$ $\equiv 1.76267477307099$. As shown in Fig. 7, sufficient accuracies were attained by the current $\mathrm{ZM}$ method, while the results of the cubic MIC were poor. Comparing with $\mathrm{CsCl}$ and $\mathrm{NaCl}$ results obtained with the ZM method, first, smaller values of $\alpha$ were preferred in the $\mathrm{CsCl}$ case. Second, a longer cutoff length was required in the $\mathrm{CsCl}$ case, as compared with the $\mathrm{NaCl}$ case, in order to ensure the same accuracy. However, this is not necessarily a fair comparison if we consider

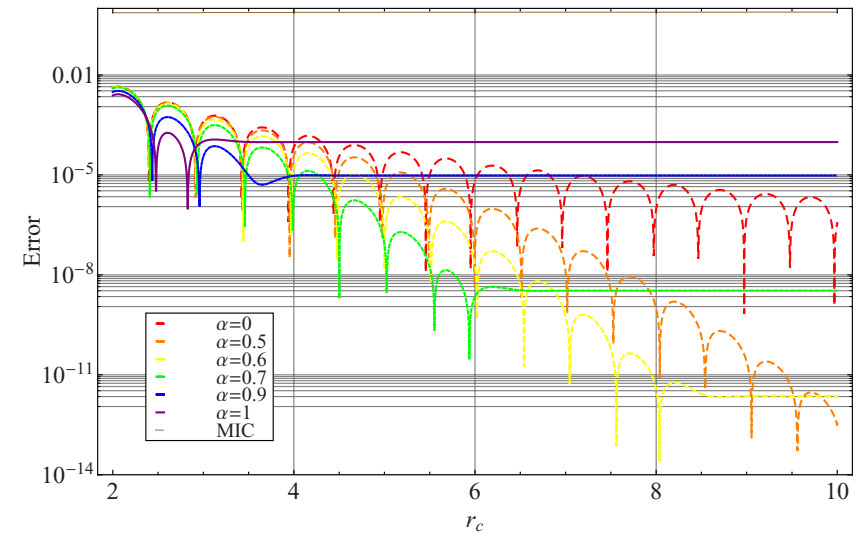

FIG. 7. The absolute value of the error (log scale) of Madelung constant of the $\mathrm{CsCl}$ crystal, obtained by the $\mathrm{ZM}$ method with moment $l=4$. Several values of the damping parameter $\alpha$ [Length $^{-1}$ ] were used. The cutoff length $r_{\mathrm{c}}$ was varied from 2 to 10 , and the unit-cell edge length was set to 1, viz., the nearest-neighbor ionic distance was $\sqrt{3} / 2$. For comparison, the results obtained by the cubic MIC method is shown by the step function, which is defined as $\Delta_{\mathrm{MIC}}\left(r_{\mathrm{c}}\right)=\Delta_{n}$ for $n \leq r_{\mathrm{c}}<n+1(n=2, \ldots, 10)$, where $\Delta_{n}$ is the absolute value of the error when the MIC utilizes $n$ unit-cells along one axis (e.g., $\Delta_{2} \sim 0.74$ ).

the actual computational cost. Based on the fact that the cost is proportional to the number of particles in the unit cell and that the number in the $\mathrm{CsCl}$ cell is one-fourth of that in the $\mathrm{NaCl}$ cell, the error obtained at $r_{\mathrm{c}}$ distance for the $\mathrm{NaCl}$ should be compared with the error at $4^{1 / 3} r_{\mathrm{c}} \sim 1.6 r_{\mathrm{c}}$ for the CsCl. In this sense, the accuracies with the same computational cost were similar between the $\mathrm{NaCl}$ and $\mathrm{CsCl}$ cases.

\section{CONCLUDING REMARKS}

A novel idea, zero-multipole summation, was proposed for evaluating the electrostatic energy of a point charge particle system in molecular simulation. By extending the ZD scheme, any higher order multipole is taken into account to characterize the neutralized subset of particles. This summation prevents the nonzero-multipole states that may be artificially generated by a simple cutoff truncation, which often causes large energetic noise. Since the ZM method with the multiple moment $l=1$ is equal to the $\mathrm{ZD}$ method, the former includes the latter. Furthermore, the energy form of the ZM method with $l=0$ is that of the ZC scheme of Wolf et al. An error analysis of these methods was performed and the intimate relationships among other non-Ewald methods were discussed. Although the derivation of the ZM method took several logical steps with mathematical proofs, the resulting energy functional form is represented in a simple pairwise fashion, allowing an easy implementation. It was clarified that the pairwise function form is unique, as long as the original pair function $V(r)$ is approximated by an even-power polynomial in the excess subset and the degree of the polynomial is equal to twice the degree of the excess-energy approximation.

In this work, the Madelung energy, or the energy for the one-snapshot crystal state, was estimated by the current method, and the efficiency was confirmed. The simplicity enables its estimation with a laptop computer. It is important to investigate the efficiencies for more complicated states, for which some numerical sequence accelerative 
transformations ${ }^{74}$ may be helpful. Another important step is to investigate the total efficiency for many snapshots of the states, which can be performed via an MD or MC calculation. Nonetheless, the computational timing for such calculations should be comparable to that of the ZD scheme previously developed, since only an even-power polynomial with respect to the atomic distance is added to the original pair function. The parallel computational timing of the ZD scheme was previously discussed, ${ }^{59,60}$ and good scalability is expected for large systems. This is a consequence of the simple pairwise energy function formula. In contrast, the application to a nonperiodic, disordered, or heterogeneous system is needed to understand the relationship between the material properties and the zero-multipole principle. This is a critical task to obtain definitive knowledge about a moment $l$ intrinsic to an arbitrary given system or a moment $l$ suitable to the system in the current method. This is also important to reveal a specific physical condition that is essentially relevant to the zeromultipole condition. In addition to the multipole moment $l$, the method includes two parameters, the cutoff length and the damping parameter. Although the relationships among the non-Ewald methods were discussed in terms of the characteristics of these parameters, more numerical investigations regarding the dependence of the efficiency on these parameters will be needed to facilitate the simulations. These important issues are now being studied, and the results will be published elsewhere.

Due to their very simplified features, CB methods are promising for long-term simulations of large systems, using high performance computing schemes. Among them, some approaches may be heuristic but effective, and other approaches may be theoretical but insufficient. Irrespective of the previously obtained presumptions, limitations, and derivations, reconsidering these approaches with novel views, analyses, and applications would be valuable for the further development of a mature method.

\section{ACKNOWLEDGMENTS}

The author thanks Professor Haruki Nakamura and Professor Shoji Takada for useful discussions. This research was supported by Research and Development of the NextGeneration Integrated Simulation of Living Matter, a part of the Development and Use of the Next-Generation Supercom- puter Project of the Ministry of Education, Culture, Sports, Science and Technology of Japan, and by a Grant-in-Aid for Scientific Research (C) (25390156) from JSPS.

\section{APPENDIX A: EXISTENCE AND UNIQUENESS OF THE COEFFICIENTS}

Fix any nonnegative integer $l$, and let $V$ be a function of class $C^{l}[V$ given by Eq. (3) applies for any $l]$. The Taylor expansion of the polynomial $V_{l}(r) \equiv \sum_{m=0}^{l} a_{m}^{(l)} r^{2 m}$ around $r=r_{\mathrm{c}}$ up to the $l$ th order yields

with

$$
V_{l}(r)=\tilde{V}_{l}(r)+o\left(\left(r-r_{\mathrm{c}}\right)^{l}\right)\left(r \rightarrow r_{\mathrm{c}}\right)
$$

$$
\tilde{V}_{l}(r) \equiv \sum_{m=0}^{l} \frac{D^{m} V_{l}\left(r_{\mathrm{c}}\right)}{m !}\left(r-r_{\mathrm{c}}\right)^{m} .
$$

If this expansion coincides with the similar expansion of $V$, i.e.,

$$
\tilde{V}_{l}(r)=\sum_{m=0}^{l} \frac{D^{m} V\left(r_{\mathrm{c}}\right)}{m !}\left(r-r_{\mathrm{c}}\right)^{m} \quad \text { for all } r>0,
$$

then we have the desired results,

$$
V(r)=V_{l}(r)+o\left(\left|r-r_{\mathrm{c}}\right|^{l}\right)\left(0<r \rightarrow r_{\mathrm{c}}\right),
$$

since

$$
\begin{aligned}
V(r) & =\tilde{V}_{l}(r)+o\left(\left(r-r_{\mathrm{c}}\right)^{l}\right) \\
& =V_{l}(r)+o\left(\left(r-r_{\mathrm{c}}\right)^{l}\right)\left(r \rightarrow r_{\mathrm{c}}\right) .
\end{aligned}
$$

The relationship in Eq. (A1) is ensured by condition (17), i.e.,

$$
D^{m} V_{l}\left(r_{\mathrm{c}}\right)=D^{m} V\left(r_{\mathrm{c}}\right) \quad \text { for } m=0, \ldots, l,
$$

which is equivalent to a linear equation with respect to $\left\{a_{m}^{(l)}\right\}_{m=0,1, \ldots, l}$ :

$$
\left[\begin{array}{c}
V\left(r_{\mathrm{c}}\right) \\
D^{1} V\left(r_{\mathrm{c}}\right) \\
D^{2} V\left(r_{\mathrm{c}}\right) \\
\vdots \\
D^{l} V\left(r_{\mathrm{c}}\right)
\end{array}\right]=A^{[l]}\left(r_{\mathrm{c}}\right)\left[\begin{array}{c}
a_{0}^{(l)} \\
a_{1}^{(l)} \\
a_{2}^{(l)} \\
\vdots \\
a_{l}^{(l)}
\end{array}\right],
$$

where $D^{n} V\left(r_{\mathrm{c}}\right)(n=0, \ldots, l)$ are given values and $A^{(l)}\left(r_{\mathrm{c}}\right)$ is an $(l+1, l+1)$-matrix defined by

$A^{(l)}\left(r_{\mathrm{c}}\right)$

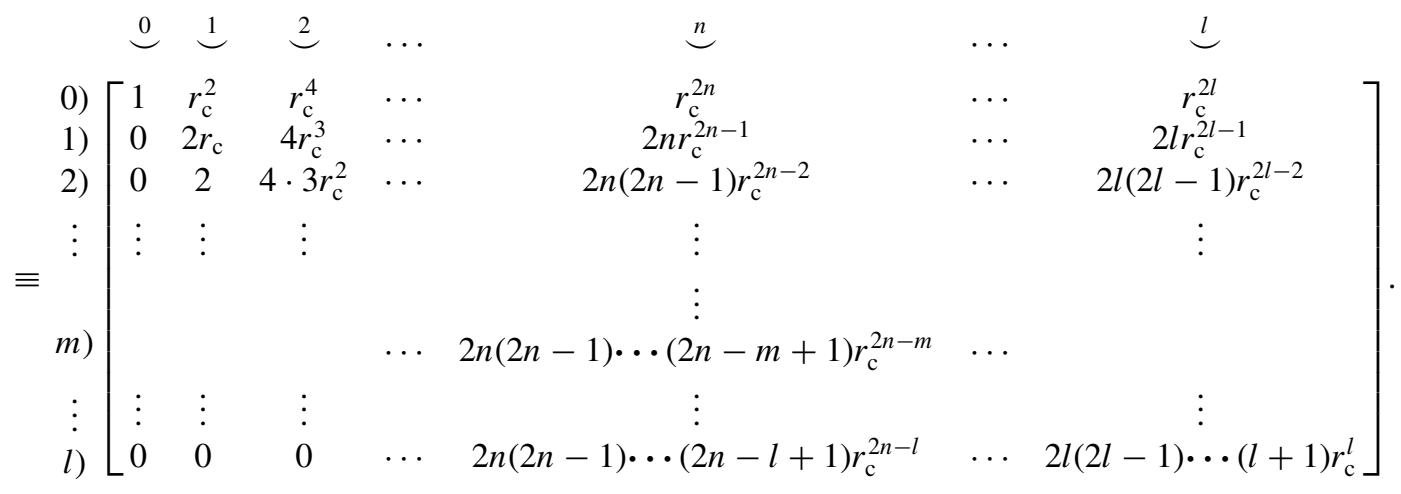


For any $l \in \mathbb{N}_{0}$ and for any $r_{\mathrm{c}}>0$, we can see

$$
\begin{aligned}
\operatorname{det} A^{(l)}\left(r_{\mathrm{c}}\right) & =\operatorname{det} A^{(l)}(1) r_{\mathrm{c}}^{\frac{l(l+1)}{2}} \\
& =2 l ! ! \cdot 2(l-1) ! ! \cdots \cdot 2 ! ! \cdot 0 ! ! r_{\mathrm{c}}^{\frac{l(l+1)}{2}} \neq 0,
\end{aligned}
$$

by noticing that elementary transformations for $A^{(l)}(1)$ yield its determinant to be the van der Monde determinant of the order $l+1, \operatorname{det}\left(\left(s_{j}\right)^{i-1}\right)$ with $s_{j} \equiv 2(j-1)$ for $i, j=1, \ldots, l$ +1 . Thus the linear equation, Eq. (A4), is solved uniquely with respect to $\left\{a_{m}^{(l)}\right\}$ for any $l$ and $r_{\mathrm{c}}$, and we can consider Eq. (A3) to always be valid.

Here we have proved that if Eq. (A3) holds, then Eq. (A2) is valid. The converse also holds. Namely, if we suppose that Eq. (A2) is valid, then the Taylor expansion of the $C^{l}$ function, $V-V_{l}$, at $r=r_{\mathrm{c}}$ leads to the result that its every $m$ th derivative vanishes at $r_{\mathrm{c}}$ for $0 \leq m \leq l$. This means Eq. (A3). Therefore, for $l \in \mathbb{N}_{0}$, we have proved that $A^{[l]}\left(r_{\mathrm{c}}\right)$ is invertible and that Eq. (A2) holds if and only if

$$
\left[\begin{array}{c}
a_{0}^{(l)} \\
a_{1}^{(l)} \\
a_{2}^{(l)} \\
\vdots \\
a_{l}^{(l)}
\end{array}\right]=A^{[l]}\left(r_{\mathrm{c}}\right)^{-1}\left[\begin{array}{c}
V\left(r_{\mathrm{c}}\right) \\
D^{1} V\left(r_{\mathrm{c}}\right) \\
D^{2} V\left(r_{\mathrm{c}}\right) \\
\vdots \\
D^{l} V\left(r_{\mathrm{c}}\right)
\end{array}\right] .
$$

\section{APPENDIX B: PROOF OF EQ. (21)}

Fix any given $x \in \Gamma$ and $i \in \mathcal{N}$, and denote the motion of the displacement vector by a map

$$
\begin{aligned}
I_{i}^{x} & \rightarrow \Gamma \subset \mathbb{R}^{d N}, \\
h^{(i)} & \mapsto x^{[i]} \equiv\left(x_{1}^{[i]}, \ldots, x_{N}^{[i]}\right) \\
& \stackrel{\mathrm{d}}{=} x^{(i)}+h^{(i)} \equiv\left(x_{1}^{(i)}+h_{1}^{(i)}, \ldots, x_{N}^{(i)}+h_{N}^{(i)}\right) .
\end{aligned}
$$

To show Eq. (21), first we see that

$$
\begin{aligned}
& \left(\hat{E}_{i}-\breve{E}_{i}\right)\left(x^{[i]}\right) \\
& =\sum_{j \in \mathcal{J}_{i}^{(l)}} q_{i} q_{j}\left(V-V_{l}\right)\left(r_{i j}^{[i]}\right),
\end{aligned}
$$

where

$$
r_{i j}^{[i]} \equiv\left\|x_{i}^{[i]}-x_{j}^{[i]}\right\|=r_{\mathrm{c}}-\left\|h_{j}^{(i)}\right\|\left(j \in \mathcal{J}_{i}^{(l)}\right) .
$$

From Eq. (16), we have

$$
u(s) \equiv V\left(r_{\mathrm{c}}-s\right)-V_{l}\left(r_{\mathrm{c}}-s\right)=o\left(|s|^{l}\right)(s \rightarrow 0),
$$

where $s \in J_{\mathrm{c}} \equiv\left(-\infty, r_{\mathrm{c}}\right)$. Note $\left\|h_{j}^{(i)}\right\|=\left\|\pi_{j}\right\|\left(h^{(i)}\right) \in\left[0, r_{\mathrm{c}}\right)$ $\subset J_{\mathrm{c}}$, with

$$
\pi_{j}: \mathbb{R}^{N d} \rightarrow \mathbb{R}^{d},\left(a_{1}, \ldots, a_{N}\right) \mapsto a_{j},
$$

being a projection. Thus for $j \in \mathcal{J}_{i}^{(l)}$,

$$
\begin{aligned}
& \left(V-V_{l}\right)\left(r_{i j}^{[i]}\right) \\
& =u\left(\left\|h_{j}^{(i)}\right\|\right) \\
& =o\left(\left\|h_{j}^{(i)}\right\|^{l}\right)\left(J_{\mathrm{c}} \ni\left\|h_{j}^{(i)}\right\| \rightarrow 0\right) .
\end{aligned}
$$

From Eqs. (B1) and (B2), considering the fact that $\left\|\pi_{j}\right\|\left(I_{i}^{x}\right) \subset J_{\mathrm{c}}$ and $u(0)=0$ [see the condition for $m=0$ in Eq. (17)], we have

$$
\begin{aligned}
\left(\hat{E}_{i}\right. & \left.-\breve{E}_{i}\right)\left(x^{[i]}\right) \\
& =\sum_{j \in \mathcal{J}_{i}} q_{i} q_{j} o\left(\left\|h_{j}^{(i)}\right\|^{l}\right)\left(J_{\mathrm{c}} \ni\left\|h_{j}^{(i)}\right\| \rightarrow 0\right) \\
& =\sum_{j \in \mathcal{J}_{i}} q_{i} q_{j} o\left(\left\|h^{(i)}\right\|^{l}\right)\left(I_{i}^{x} \ni h^{(i)} \rightarrow 0\right) \\
& =o\left(\left\|h^{(i)}\right\|^{l}\right),
\end{aligned}
$$

where we have used the Euclidian norms on both $\left\|h_{j}^{(i)}\right\|$ for $h_{j}^{(i)} \in \mathbb{R}^{d}$ and $\left\|h^{(i)}\right\|$ for $h^{(i)} \in \mathbb{R}^{d N}$.

APPENDIX C: PROOF OF EQ. (27)

Fix an arbitrary $m \in\{0, \ldots, l\}$. By expanding $r_{i j}^{2 m}$, we get

$$
\begin{aligned}
& \sum_{i \in \mathcal{N}} \sum_{j \in \mathcal{J}_{i}^{(l)}} q_{i} q_{j} r_{i j}^{2 m} \\
& =\sum_{i \in \mathcal{N}} \sum_{j \in \mathcal{J}_{i}^{(l)}} q_{i} q_{j}\left[\left\|x_{i}\right\|^{2}+\left\|x_{j}\right\|^{2}-2\left(x_{i} \mid x_{j}\right)\right]^{m} \\
& =\sum_{n=0}^{m} \sum_{k=0}^{n} \sum_{i \in \mathcal{N}} \sum_{j \in \mathcal{J}_{i}^{(l)}} a_{i j n k} \\
& =\sum_{(n, k) \in G_{m}} \sum_{i \in \mathcal{N}} \sum_{j \in \mathcal{J}_{i}^{(l)}} a_{i j n k}+\sum_{(n, k) \in G_{m}^{c}} \sum_{i \in \mathcal{N}} \sum_{j \in \mathcal{J}_{i}^{(l)}} a_{i j n k},
\end{aligned}
$$

where

$$
\begin{aligned}
a_{i j n k} & \equiv\left(\begin{array}{c}
m \\
n k
\end{array}\right)(-2)^{k} q_{i} q_{j}\left(x_{i} \mid x_{i}\right)^{m-n}\left(x_{j} \mid x_{j}\right)^{n-k}\left(x_{i} \mid x_{j}\right)^{k}, \\
\left(\begin{array}{c}
m \\
n k
\end{array}\right) & \equiv \frac{m !}{(m-n) !(n-k) ! k !} .
\end{aligned}
$$

Here, to efficiently use condition (5b), we have introduced subsets,

$$
G_{m} \equiv\{(n, k) \mid k \in\{0, \ldots, n\}, n \in\{0, \ldots, m\}, 2 n-k \leq m\}
$$

and

$$
G_{m}^{c} \equiv\{(n, k) \mid k \in\{0, \ldots, n\}, n \in\{0, \ldots, m\}, 2 n-k>m\} .
$$

Now, to evaluate Eq. (C1d), we observe

$$
\sum_{j \in \mathcal{J}_{i}^{(l)}} q_{j}\left(x_{j} \mid x_{j}\right)^{n-k}\left(x_{i} \mid x_{j}\right)^{k}
$$

$$
\begin{aligned}
= & \sum_{j \in \mathcal{J}_{i}^{(l)}} q_{j} \sum_{\alpha_{1}=1}^{d} \cdots \sum_{\alpha_{n-k}=1}^{d} x_{j, \alpha_{1}}^{2} \cdots \cdots x_{j, \alpha_{n-k}}^{2} \\
& \times \sum_{\beta_{1}=1}^{d} \cdots \sum_{\beta_{k}=1}^{d} x_{i, \beta_{1}} \cdots x_{i, \beta_{k}} x_{j, \beta_{1}} \cdots x_{j, \beta_{k}}
\end{aligned}
$$




$$
\begin{aligned}
= & \sum_{\beta_{1}=1}^{d} \cdots \sum_{\beta_{k}=1}^{d} x_{i, \beta_{1}} \cdots \cdots x_{i, \beta_{k}} \\
& \times \sum_{\alpha_{1}=1}^{d} \cdots \sum_{\alpha_{n-k}=1}^{d} \sum_{j \in \mathcal{J}_{i}^{(l)}} q_{j} x_{j, \alpha_{1}}^{2} \cdots \cdots x_{j, \alpha_{n-k}}^{2} x_{j, \beta_{1}} \cdots x_{j, \beta_{k}} .
\end{aligned}
$$

We can easily represent the results for even the $n-k=0$ or $k=0$ case from Eq. $(\mathrm{C} 2 \mathrm{c})$.

Here, if $(n, k) \in G_{m}$ (note $G_{m}$ is always non-empty), then the summation with respect to $j$ can be performed by using the following relationship, derived from Eqs. (5a) and (5b), for a zero $m$ th-moment condition:

$$
\begin{aligned}
& \sum_{j \in \mathcal{J}_{i}^{(l)}} q_{j} x_{j, \alpha_{1}} \cdots \cdots x_{j, \alpha_{m}} \\
& \quad=\sum_{j \in \mathcal{R}^{i}} q_{j} x_{j, \alpha_{1}} \cdots x_{j, \alpha_{m}}, 0 \leq m \leq l,
\end{aligned}
$$

for any $\alpha_{1}, \ldots, \alpha_{m} \in\{1, \ldots, d\}$. This is because the total number of coordinate components $x_{j, \alpha}$ arising in the product in the summation $\sum_{j \in \mathcal{J}_{i}^{(l)}}$ in Eq. (C2c) is $0 \leq 2(n-k)+k$ $=2 n-k \leq m \leq l$. Thus,

$$
\begin{aligned}
& \sum_{j \in \mathcal{J}_{i}^{(l)}} q_{j} x_{j, \alpha_{1}}^{2} \cdots \cdots x_{j, \alpha_{n-k}}^{2} x_{j, \beta_{1}} \cdots x_{j, \beta_{k}} \\
& \quad=\sum_{j \in \mathcal{R}^{i}} q_{j} x_{j, \alpha_{1}}^{2} \cdots x_{j, \alpha_{n-k}}^{2} x_{j, \beta_{1}} \cdots x_{j, \beta_{k}},
\end{aligned}
$$

and so

$$
\sum_{j \in \mathcal{J}_{i}^{(l)}} q_{j}\left(x_{j} \mid x_{j}\right)^{n-k}\left(x_{i} \mid x_{j}\right)^{k}=\sum_{j \in \mathcal{R}^{i}} q_{j}\left(x_{j} \mid x_{j}\right)^{n-k}\left(x_{i} \mid x_{j}\right)^{k},
$$

leading to

$$
\sum_{(n, k) \in G_{m}} \sum_{i \in \mathcal{N}} \sum_{j \in \mathcal{J}_{i}^{(l)}} a_{i j n k}=\sum_{(n, k) \in G_{m}} \sum_{i \in \mathcal{N}} \sum_{j \in \mathcal{R}^{i}} a_{i j n k} .
$$

Unfortunately, if $(n, k) \in G_{m}^{c}$ (except for $m=0$ ), then it happens to become $2 n-k>l$. However, the consistency condition (10) allows us to proceed. In fact, first, according to this condition, we have

$$
\sum_{(n, k) \in G_{m}^{c}} \sum_{i \in \mathcal{N}} \sum_{j \in \mathcal{J}_{i}^{(l)}} a_{i j n k}=\sum_{(n, k) \in G_{m}^{c}} \sum_{i \in \mathcal{N}} \sum_{j \in \mathcal{J}_{i}^{(l)}} a_{j i n k},
$$

where

$$
a_{j i n k}=\left(\begin{array}{c}
m \\
n k
\end{array}\right)(-2)^{k} q_{i} q_{j}\left(x_{i} \mid x_{i}\right)^{n-k}\left(x_{j} \mid x_{j}\right)^{m-n}\left(x_{i} \mid x_{j}\right)^{k} .
$$

Second, when we expand $\sum_{j \in \mathcal{J}_{i}^{(l)}} q_{j}\left(x_{j} \mid x_{j}\right)^{m-n}\left(x_{i} \mid x_{j}\right)^{k}$, as done in Eq. (C2), the total number of coordinate components in the summation $\sum_{j \in \mathcal{J}_{i}^{(l)}}$ is $0 \leq 2(m-n)+k=2 m-(2 n$ $-k)<m \leq l$. Thus, similar to the situation above, we obtain

$$
\sum_{(n, k) \in G_{m}^{c}} \sum_{i \in \mathcal{N}} \sum_{j \in \mathcal{J}_{i}^{(l)}} a_{i j n k}=\sum_{(n, k) \in G_{m}^{c}} \sum_{i \in \mathcal{N}} \sum_{j \in \mathcal{R}^{i}} a_{j i n k} .
$$

Since it is clear that $\sum_{i \in \mathcal{N}} \sum_{j \in \mathcal{R}^{i}} a_{j i n k}=\sum_{i \in \mathcal{N}} \sum_{j \in \mathcal{R}^{i}} a_{i j n k}$, we get

$$
\begin{aligned}
& \sum_{i \in \mathcal{N}} \sum_{j \in \mathcal{J}_{i}^{(l)}} q_{i} q_{j} r_{i j}^{2 m} \\
& =\sum_{(n, k) \in G_{m}} \sum_{i \in \mathcal{N}} \sum_{j \in \mathcal{J}_{i}^{(l)}} a_{i j n k}+\sum_{(n, k) \in G_{m}^{c}} \sum_{i \in \mathcal{N}} \sum_{j \in \mathcal{J}_{i}^{(l)}} a_{i j n k} \\
& =\sum_{(n, k) \in G_{m}} \sum_{i \in \mathcal{N}} \sum_{j \in \mathcal{R}^{i}} a_{i j n k}+\sum_{(n, k) \in G_{m}^{c}} \sum_{i \in \mathcal{N}} \sum_{j \in \mathcal{R}^{i}} a_{i j n k} \\
& =\sum_{i \in \mathcal{N}} \sum_{j \in \mathcal{R}^{i}} q_{i} q_{j} r_{i j}^{2 m} .
\end{aligned}
$$

${ }^{1}$ P. J. Steinbach and B. R. Brooks, J. Comput. Chem. 15, 667 (1994).

${ }^{2}$ P. P. Ewald, Ann. Phys. (Leipzig) 64, 253 (1921).

${ }^{3}$ D. Borwein, J. M. Borwein, and K. F. Taylor, J. Math. Phys. 26, 2999 (1985).

${ }^{4}$ S. W. de Leeuw, J. W. Perram, and E. R. Smith, Proc. R. Soc. London, Ser. A 373, 27 (1980).

${ }^{5}$ C. Sagui and T. A. Darden, Annu. Rev. Biophys. Biomol. Struct. 28, 155 (1999).

${ }^{6}$ F. E. Harris and H. J. Monkhorst, Phys. Rev. B 2, 4400 (1970).

${ }^{7}$ R. E. Crandall and J. F. Delord, J. Phys. A 20, 2279 (1987).

${ }^{8}$ R. Sperb, Mol. Simul. 22, 199 (1999).

${ }^{9}$ A. Hautot, J. Phys. A 8, 853 (1975).

${ }^{10}$ J. Lekner, Physica A 176, 485 (1991).

${ }^{11}$ S. Tyagi, Phys. Rev. E 70, 066703 (2004).

${ }^{12}$ S. Tyagi, Prog. Theor. Phys. 114, 517 (2005).

${ }^{13}$ A. D. Baker, M. D. Baker, and C. R. H. Hanusa, J. Math. Chem. 49, 1192 (2011).

${ }^{14}$ I. Fukuda and H. Nakamura, Biophys. Rev. 4, 161 (2012).

${ }^{15}$ L. Onsager, J. Am. Chem. Soc. 58, 1486 (1936).

${ }^{16}$ J. A. Barker and R. O. Watts, Mol. Phys. 26, 789 (1973).

${ }^{17}$ O. Steinhauser, Mol. Phys. 45, 335 (1982).

${ }^{18}$ P. H. Hünenberger and W. F. van Gunsteren, J. Chem. Phys. 108, 6117 (1998).

${ }^{19}$ R. Gargallo, P. H. Hünenberger, F. X. Avilés, and B. Oliva, Protein Sci. 12, 2161 (2003)

${ }^{20}$ A. Robertson, E. Luttmann, and V. S. Pande, J. Comput. Chem. 29, 694 (2008).

${ }^{21}$ E. Yakub and C. Ronchi, J. Chem. Phys. 119, 11556 (2003).

${ }^{22}$ E. Yakub and C. Ronchi, J. Low Temp. Phys. 139, 633 (2005).

${ }^{23}$ E. S. Yakub, J. Phys. A 39, 4643 (2006).

${ }^{24}$ P. K. Jha, R. Sknepnek, G. I. Guerrero-García, and M. Olvera de la Cruz, J. Chem. Theory Comput. 6, 3058 (2010).

${ }^{25}$ G. Vernizzi, G. I. Guerrero-García, and M. Olvera de la Cruz, Phys. Rev. E 84, 016707 (2011).

${ }^{26} \mathrm{X}$. Wu and B. R. Brooks, J. Chem. Phys. 122, 044107 (2005).

${ }^{27}$ X. Wu and B. R. Brooks, J. Chem. Phys. 131, 024107 (2009).

${ }^{28}$ I. H. Hristov, R. Paul, and S. J. Paddison, J. Chem. Phys. 131, 164103 (2009).

${ }^{29}$ K. Z. Takahashi, T. Narumi, and K. Yasuoka, J. Chem. Phys. 135, 174108 (2011).

${ }^{30}$ R. W. Pastor and A. D. MacKerell, Jr., J. Phys. Chem. Lett. 2, 1526 (2011).

${ }^{31}$ X. Wu and B. R. Brooks, J. Chem. Phys. 129, 154115 (2008).

${ }^{32}$ D. Wolf, Phys. Rev. Lett. 68, 3315 (1992).

${ }^{33}$ D. Wolf, P. Keblinski, S. R. Phillpot, and J. Eggebrecht, J. Chem. Phys. 110, 8254 (1999).

${ }^{34}$ D. Zahn, B. Schilling, and S. M. Kast, J. Phys. Chem. B 106, 10725 (2002).

${ }^{35}$ C. Avendaño and A. Gil-Villegas, Mol. Phys. 104, 1475 (2006).

${ }^{36}$ C. J. Fennell and J. D. Gezelter, J. Chem. Phys. 124, 234104 (2006).

${ }^{37}$ M. Sepliarsky, M. G. Stachiotti, and R. L. Migoni, Phys. Rev. Lett. 96, 137603 (2006).

${ }^{38}$ M. C. C. Ribeiro, Phys. Rev. B 75, 144202 (2007)

${ }^{39}$ T. G. Desai, J. Chem. Phys. 127, 154707 (2007).

${ }^{40}$ M. Goto, H. Takezoe, and K. Ishikawa, Phys. Rev. E 76, 040701(R) (2007).

${ }^{41}$ T. S. Mahadevan and S. H. Garofalini, J. Phys. Chem. B 111, 8919 (2007). 
${ }^{42}$ D. S. Aidhy, P. C. Millett, T. Desai, D. Wolf, and S. R. Phillpot, Phys. Rev. B 80, 104107 (2009).

${ }^{43}$ Y. Nagata and S. Mukamel, J. Am. Chem. Soc. 132, 6434 (2010).

${ }^{44}$ D.-L. Chen, A. C. Stern, B. Space, and J. K. Johnson, J. Phys. Chem. A 114, 10225 (2010).

${ }^{45}$ S. Kuang and J. D. Gezelter, J. Chem. Phys. 133, 164101 (2010).

${ }^{46}$ E. E. Gdoutos, R. Agrawal, and H. D. Espinosa, Int. J. Numer. Methods Eng. 84, 1541 (2010).

${ }^{47}$ G. Jiménez-Serratos, C. Avendaño, A. Gil-Villegas, and E. GonzálezTovar, Mol. Phys. 109, 27 (2011).

${ }^{48}$ Z. Cui, Y. Sun, Y. Chen, and J. Qu, Solid State Ionics 187, 8 (2011).

${ }^{49}$ P. X. Viveros-Méndez and A. Gil-Villegas, J. Chem. Phys. 136, 154507 (2012).

${ }^{50}$ Y. Wang and X. Xu, Appl. Phys. A 110, 617 (2013).

${ }^{51}$ B. Guchhait, R. Biswas, and P. K. Ghorai, J. Phys. Chem. B 117, 3345 (2013).

${ }^{52}$ S. Dai and H. S. Park, J. Mech. Phys. Solids 61, 385 (2013).

${ }^{53}$ J. A. Gee, J. Chung, S. Nair, and D. S. Sholl, J. Phys. Chem. C 117, 3169 (2013).

${ }^{54}$ S. Yang, L. Xiong, Q. Deng, and Y. Chen, Acta Mater. 61, 89 (2013).

${ }^{55}$ B. Deng, A. Chernatynskiy, P. Shukla, S. B. Sinnott, and S. R. Phillpot, J. Nucl. Mater. 434, 203 (2013).

${ }^{56}$ I. Fukuda, Y. Yonezawa, and H. Nakamura, J. Phys. Soc. Jpn. 77, 114301 (2008).

${ }^{57}$ Y. Yonezawa, I. Fukuda, N. Kamiya, H. Shimoyama, and H. Nakamura, J. Chem. Theory Comput. 7, 1484 (2011).

${ }^{58}$ I. Fukuda, Y. Yonezawa, and H. Nakamura, J. Chem. Phys. 134, 164107 (2011).
${ }^{59}$ I. Fukuda, N. Kamiya, Y. Yonezawa, and H. Nakamura, J. Chem. Phys. 137, 054314 (2012).

${ }^{60}$ N. Kamiya, I. Fukuda, and H. Nakamura, Chem. Phys. Lett. 568-569, 26 (2013).

${ }^{61}$ The domain $I_{i}^{x}$ can be defined in the same manner as done in Appendix A of Ref. 58, with a replacement $\mathcal{J}_{i} \rightarrow \mathcal{J}_{i}^{(l)}$. Conditions of $I_{i}^{x}$ for the consistent descriptions are also currently satisfied. This can be shown by notifying, especially, the fact that for $x \in \Gamma_{l}$, assigning $\mathcal{M}_{i}^{(l)}\left(x^{[i]}\right) \equiv \mathcal{M}_{i}^{(l)}(x)$ with $x^{[i]}$ $\equiv x^{(i)}+h^{(i)}$ for all $h^{(i)} \in I_{i}^{x}$ enables us to retain the algebraic ZM condition: $\sum_{j \in \mathcal{M}_{i}^{(l)}\left(x^{[i]}\right) \cup\{i\}} q_{j} x_{j}^{[i]} \otimes . \stackrel{m}{.} \otimes x_{j}^{[i]}=\sum_{j \in \mathcal{M}_{i}^{(l)}(x) \cup\{i\}} q_{j} x_{j} \otimes \stackrel{m}{*} \otimes$ $x_{j}=0$.

${ }^{62}$ W. A. Harrison, Phys. Rev. B 73, 212103 (2006).

${ }^{63}$ D. van der Spoel and P. J. van Maaren, J. Chem. Theory Comput. 2, 1 (2006).

${ }^{64}$ A. Baumketner, J. Chem. Phys. 130, 104106 (2009).

${ }^{65}$ G. I. Guerrero-García, P. González-Mozuelos, and M. Olvera de la Cruz, J. Chem. Phys. 135, 164705 (2011).

${ }^{66}$ I. V. Abarenkov, Phys. Rev. B 76, 165127 (2007).

${ }^{67}$ E. Madelung, Phys. Z. 19, 524 (1918).

${ }^{68}$ V. Drchal, R. Hammerling, and P. Weinberger, Phys. Rev. B 74, 214202 (2006).

${ }^{69}$ I. Hase and T. Yanagisawa, Phys. Rev. B 76, 174103 (2007).

${ }^{70}$ R. E. Crandall, Exp. Math. 8, 367 (1999).

${ }^{71}$ S. G. Brush, H. L. Sahlin, and E. Teller, J. Chem. Phys. 45, 2102 (1966).

${ }^{72}$ A. Angoshtari and A. Yavari, Phys. Lett. A 375, 1281 (2011).

${ }^{73}$ Y. Sakamoto, J. Chem. Phys. 28, 164 (1958).

${ }^{74}$ B. Sarkar and K. Bhattacharyya, Phys. Rev. B 48, 6913 (1993). 